REPRESENTATION THEORY

An Electronic Journal of the American Mathematical Society

Volume 8, Pages 15-51 (April 15, 2004)

S $1088-4165(04) 00215-8$

\title{
RESOLUTIONS AND HILBERT SERIES OF THE UNITARY HIGHEST WEIGHT MODULES OF THE EXCEPTIONAL GROUPS
}

\author{
THOMAS J. ENRIGHT AND MARKUS HUNZIKER
}

\begin{abstract}
We give a sufficient criterion on a highest weight module of a semisimple Lie algebra to admit a resolution in terms of sums of modules induced from a parabolic subalgebra. In particular, we show that all unitary highest weight modules admit such a resolution. As an application of our results we compute (minimal) resolutions and explicit formulas for the Hilbert series of the unitary highest weight modules of the exceptional groups.
\end{abstract}

\section{INTRODUCTION}

1.1. Bernstein, Gelfand and Gelfand $[\mathrm{BGG}$, gave a resolution of each finite dimensional representation $F$ of a semisimple Lie algebra $\mathfrak{g}$ in terms of sums of representations induced from one-dimensional representations of a Borel subalgebra (the Verma modules). This result was extended by Lepowsky $[\underline{\mathrm{L}}$ to give resolutions of finite dimensional representations in terms of sums of representations induced from parabolic subalgebras (the generalized Verma modules). In this article we extend these results by replacing the finite dimensional representation $F$ by an irreducible highest weight representation $L$. We give a sufficient criterion on $L$ for such a resolution to exist. Our criterion takes the form of a condition on the $\mathfrak{u}$-cohomology of $L$, where $\mathfrak{u}$ is the nilradical of the parabolic subalgebra. These resolutions we will call generalized BGG resolutions.

1.2. As an application of our results we turn to the exceptional groups with unitary highest weight representations. We prove that every unitary highest weight representation has a generalized BGG resolution. For classical groups this result is in [EW]. We note that in the case of unitary highest weight representations, i.e., in the Hermitian symmetric setting, the BGG resolutions can be interpreted as graded free resolutions in the sense of commutative algebra as follows. Let $(G, K)$ be an irreducible Hermitian symmetric pair and let $(\mathfrak{g}, \mathfrak{k})$ be the corresponding pair of complexified Lie algebras. We have the usual decomposition $\mathfrak{g}=\mathfrak{k} \oplus \mathfrak{p}^{+} \oplus \mathfrak{p}^{-}$of $\mathfrak{g}$ as a $\mathfrak{k}^{-}$-module. Let $S=S\left(\mathfrak{p}^{-}\right)$be the symmetric algebra of $\mathfrak{p}^{-}$. Then every highest weight module $L$ of $(\mathfrak{g}, \mathfrak{k})$ is also a finitely generated graded $S$-module. As such, $L$ admits a minimal free resolution. It was proved in $[\mathrm{EH}]$ by the authors of this paper that the generalized BGG resolutions of unitary highest weight representations are minimal free resolutions.

Received by the editors October 22, 2003.

2000 Mathematics Subject Classification. Primary 22E47, 17B10, 14M12, 13 D02.

Key words and phrases. Highest weight modules, minimal resolutions, Hilbert series. 
Furthermore, we can associate a Hilbert series $h_{L}(t)$ to the graded $S$-module $L$. It is standard that $h_{L}(t)$ can be written as a rational function in the form

$$
h_{L}(t)=\frac{P(t)}{(1-t)^{d}},
$$

where $P(t)$ is polynomial with integer coefficients such that $P(1) \neq 0$. The number $d$ is equal to the Gelfand-Kirillov dimension of $L$ and the number $P(1)$ is equal to the Bernstein degree of $L$. In this paper we also compute explicitly the Hilbert series of the unitary highest weight representations of the exceptional groups.

1.3. Let $\mathfrak{h}$ be a Cartan subalgebra of $\mathfrak{k}$ and hence also $\mathfrak{g}$. In DES it was observed that the set of all $\lambda \in \mathfrak{h}^{*}$ that arise as a highest weight of some unitary highest weight representation of $\mathfrak{g}$ that is not induced (from a finite dimensional representation of the parabolic subalgebra $\mathfrak{k} \oplus \mathfrak{p}^{+}$) can be written as a disjoint union of translated integral cones. This is called the cone decomposition. In this paper we propose a finer decomposition by subdividing each cone into what we call unitary strata. We show, by using Zuckerman translation, that the Gelfand-Kirillov dimension as well as other indices associated with the generalized BGG resolution of a unitary highest weight representation $L$ with highest weight $\lambda$ are constant for $\lambda$ varying over a single stratum.

In this paper we determine explicitly all unitary strata for the exceptional groups. It turns out that there are 17 unitary strata for type $E_{6}$ and 20 unitary strata for type $E_{7}$. Each unitary stratum has a unique minimal vertex. We explicitly compute the generalized BGG resolution and the Hilbert series of the unitary highest weight representation corresponding to these vertices. The variety of numerator polynomials $P(t)$ of the Hilbert series that arise is quite surprising. Of the 37 polynomials only 6 have nonnegative coefficients and five of these lie in the Wallach set and two are the trivial representations of $E_{6}$ and $E_{7}$.

1.4. Organization of the paper. In section 2 we recall basic definitions and the generalized BGG resolution due to Lepowsky [L] for finite dimensional representations. Following this we consider hypotheses under which a highest weight representation (not necessarily finite dimensional) admits a generalized BGG resolution. In section 3 we summarize what is known about resolutions of unitary highest weight representations. We then extend these results to the setting of this article to prove that all unitary highest weight representations admit a generalized BGG resolution. In section 4 we recall the cone decomposition from [DES] and introduce the notion of unitary strata. The section ends with tables describing some of the invariants associated with the stratified cone decomposition for $E_{6}$ and $E_{7}$. In section 5 several examples are given to indicate how various parameter root subspaces are calculated. In sections 6 and 7 explicit formulas for the generalized BGG resolutions and the Hilbert series of the unitary highest weight representations are given for vertices of the unitary strata for $E_{6}$ and $E_{7}$.

1.5. Remarks and acknowledgements. This article may be regarded as an extension of the article [EH] and as such the overlap of topics with [NOTYK] applies here as well. In particular, for the Wallach representations the GelfandKirillov dimension and the Bernstein degree are computed in NOTYK. However, the full Hilbert series are not determined there. We also thank Nolan Wallach for several helpful discussions regarding cohomology and characters. 


\section{Generalized BGG Resolutions}

2.1. In this section we recall some basic definitions and constructions related to highest weight modules that will be used throughout the rest of the paper. In particular, we recall the generalized BGG resolution of a finite dimensional representation by generalized Verma modules. Then we consider the corresponding results resolving highest weight representations which are not finite dimensional.

2.2. Notation. We consider the general setting of a parabolic subalgebra of $\mathfrak{g}$. Let $\mathfrak{h}$ be a Cartan subalgebra of $\mathfrak{g}$ and let $\Delta$ be the root system of the pair $(\mathfrak{g}, \mathfrak{h})$. For $\alpha \in \Delta$, let $\mathfrak{g}_{\alpha}$ be the $\alpha$-root subspace of $\mathfrak{g}$. Let $S$ be a proper subset of the simple roots of a positive system $\Delta^{+}$of $\Delta$. Let $\Delta_{S}$ equal the subroot system generated by $S$. Let $\Delta_{S}^{+}=\Delta_{S} \cap \Delta^{+}$and write $\Delta^{+}$as a disjoint union $\Delta^{+}=\Delta_{S}^{+} \cup \Delta_{\mathfrak{u}}$. Put $\mathfrak{u}^{ \pm}=\sum_{\alpha \in \Delta_{\mathfrak{u}}} \mathfrak{g}_{ \pm \alpha}$ and $\mathfrak{r}=\mathfrak{h} \oplus \sum_{\alpha \in \Delta_{S}} \mathfrak{g}_{\alpha}$. Then $\mathfrak{q}=\mathfrak{r} \oplus \mathfrak{u}^{+}$is the parabolic subalgebra associated with $S$ and $\mathfrak{g}=\mathfrak{u}^{-} \oplus \mathfrak{q}$.

Let $W$ be the Weyl group of the pair $(\mathfrak{g}, \mathfrak{h})$ and let $W_{S}$ be the Weyl group of the pair $(\mathfrak{r}, \mathfrak{h})$ which we may identify with the subgroup of $W$ that is generated by the reflections $s_{\alpha}$ with $\alpha \in \Delta_{S}$. Let $\rho=\frac{1}{2} \sum_{\alpha \in \Delta^{+}} \alpha$ and define

$$
W^{S}=\left\{w \in W \mid w \rho \text { is } \Delta_{S}^{+} \text {-dominant }\right\}=\left\{w \in W \mid w \Delta^{+} \supset \Delta_{S}^{+}\right\} .
$$

Then $W=W_{S} \cdot W^{S}$ and $W_{S} \cap W^{S}=\{e\}$. In particular, $W^{S}$ is a set of coset representatives for $W_{S} \backslash W$. Let $\ell$ denote the usual length function on $W$.

Fix a dominant element $\mu \in \mathfrak{h}^{*}$ and define $T$ as the subset of all simple roots $\alpha$ for which $(\mu, \alpha)=0$. Define $\Delta_{T}, \Delta_{T}^{+}$and $W_{T}$ as above with $S$ replaced by $T$. The involution $x \rightarrow x^{-1}$ changes the Weyl group decomposition from right cosets to left cosets giving

$$
{ }^{T} W=\left\{w \in W \mid w^{-1} \rho \text { is } \Delta_{T}^{+} \text {-dominant }\right\}=\left\{w \in W \mid w^{-1} \Delta^{+} \supset \Delta_{T}^{+}\right\} .
$$

Then $W={ }^{T} W \cdot W_{T}$ and $W_{T} \cap{ }^{T} W=\{e\}$. In particular, ${ }^{T} W$ is a set of coset representatives for $W / W_{T}$.

2.3. Generalized Verma modules. Let $\lambda \in \mathfrak{h}^{*}$ be $\Delta_{S}^{+}$-dominant integral and let $F_{\lambda}$ be the irreducible finite dimensional $\mathfrak{r}$-module with highest weight $\lambda$. We may consider $F_{\lambda}$ as a $\mathfrak{q}=\mathfrak{q}_{S}=\mathfrak{r} \oplus \mathfrak{u}^{+}$-module by letting $\mathfrak{u}^{+}$act by zero. We then define the generalized Verma module $N_{\lambda}$ with highest weight $\lambda$ as

$$
N_{\lambda}=U(\mathfrak{g}) \otimes_{U(\mathfrak{q})} F_{\lambda} .
$$

Here $U(\mathfrak{g})$ and $U(\mathfrak{q})$ denote the universal enveloping algebras of $\mathfrak{g}$ and $\mathfrak{q}$, respectively. Let $L_{\lambda}$ denote the unique irreducible quotient of $N_{\lambda}$. By the PBW theorem

$$
N_{\lambda} \simeq S\left(\mathfrak{u}^{-}\right) \otimes F_{\lambda} \quad \text { as an } \mathfrak{r} \text {-module } .
$$

Here as $\mathfrak{r}$-modules we have identified the universal enveloping algebra $U\left(\mathfrak{u}^{-}\right)$with the symmetric algebra $S\left(\mathfrak{u}^{-}\right)$. Sometimes we will also write $N_{\mathfrak{g}, \lambda}$ and $F_{\mathfrak{r}, \lambda}$ instead of $N_{\lambda}$ and $F_{\lambda}$.

2.4. The relative category $\mathcal{O}_{S}$. We denote by $\mathcal{O}_{S}=\mathcal{O}\left(\mathfrak{g}, \mathfrak{q}_{S}\right)$ the category of $\mathfrak{g}$-modules $M$ that satisfy the following three conditions:

(i) $M$ is finitely generated as a $U(\mathfrak{g})$-module,

(ii) $M$ is locally finite as a $U(\mathfrak{q})$-module,

(iii) $M$ is completely reducible as a $U(\mathfrak{r})$-module. 
Let $\chi_{\lambda+\rho}$ denote the infinitesimal character of $N_{\lambda}$. We denote by $\mathcal{O}(\mathfrak{g}, \mathfrak{q} S)_{\lambda+\rho}$ the full subcategory of $\mathcal{O}\left(\mathfrak{g}, \mathfrak{q}_{S}\right)$ consisting of modules with generalized infinitesimal character $\chi_{\lambda+\rho}$. The simple modules in the category $\mathcal{O}\left(\mathfrak{g}, \mathfrak{q}_{S}\right)_{\lambda+\rho}$ are parametrized by a special collection of double cosets which we now define.

2.5. Double cosets and highest weights. Choose $\mu$ dominant with $\lambda+\rho \in W \mu$ and let $T$ be defined as in 2.2. Then $W / W_{T}$ parametrizes the $W$-orbit of $\mu$. For $x \in W$, the module $N_{x \mu-\rho}$ is nonzero if and only if $x \mu-\rho$ is $\Delta_{S}^{+}$-dominant integral if and only if $x \mu$ is $\Delta_{S}^{+}$-regular dominant integral. Consider the double cosets $W_{S} \backslash W / W_{T}$. Then our category $\mathcal{O}\left(\mathfrak{g}, \mathfrak{q}_{S}\right)_{\mu}$ is nonempty precisely when there exist double cosets of maximal size, $\left|W_{S}\right|\left|W_{T}\right|$. We shall assume that this is the case. Set $\mathcal{D}$ equal to the set of all such double cosets of maximal size. Each double coset $\delta \in \mathcal{D}$ contains a unique element $x$ of minimal length and we write $\delta=W_{S} x W_{T}$. Let $\delta_{x}$ denote this double coset. These elements $x$ lie in ${ }^{T} W \cap W^{S}$. Let $D$ denote the subset of ${ }^{T} W \cap W^{S}$ which is the image of $\mathcal{D}$ under $\delta_{x} \rightarrow x$. Summarizing we have:

Lemma. The highest weights of the irreducible modules in $\mathcal{O}\left(\mathfrak{g}, \mathfrak{q}_{S}\right)_{\lambda+\rho}$ are the elements $x \mu-\rho$, for $x \in D$.

2.6. The generalized BGG resolution. Define $W^{S, i}=\left\{w \in W^{S} \mid \ell(w)=i\right\}$, where $\ell$ denotes the usual length function of $W$.

Theorem $\left[\mathrm{L}\right.$. Let $\lambda \in \mathfrak{h}^{*}$ be $\Delta^{+}$-dominant integral and let $E_{\lambda}$ be the irreducible finite dimensional $\mathfrak{g}$-module with highest weight $\lambda$. Then $E_{\lambda}$ has a resolution as a $\mathfrak{g}$-module of the form

$$
0 \rightarrow N_{p} \rightarrow \cdots \rightarrow N_{1} \rightarrow N_{0} \rightarrow E_{\lambda} \rightarrow 0
$$

where

$$
N_{i}=\bigoplus_{w \in W^{S, i}} N_{w(\lambda+\rho)-\rho} .
$$

2.7. Generalized Kostant modules. The resolution given in Theorem 2.6 is our model. We wish to generalize the result by replacing $E_{\lambda}$ by an arbitrary unitary irreducible highest weight representation. Note that when the infinitesimal character is regular, the setup is more transparent than in the general case. The reader may wish to restrict to this case for a first reading.

Fix an irreducible $\mathfrak{g}$-module $L$ having infinitesimal character $\chi_{\mu}$ with $\mu$ dominant. Let $D$ be the set that was defined in 2.5. Suppose $X=\bigcup_{0 \leq i \leq t} X^{i}$ is a graded subset of $D$. We call the grading a Kostant grading if for every $1 \leq i \leq t$, any two distinct elements $x, y \in X^{i} \subseteq D$ are not comparable in the Bruhat order, i.e., $x \nprec y$ and $y \nprec x$. For $0 \leq i \leq t$, set $N_{i}=\bigoplus_{w \in X^{i}} N_{w \mu-\rho}$. We say that $L$ is a generalized Kostant module for data $X$ if the following hold:

(i) $X \subset D$ has a Kostant grading $X=\bigcup_{0 \leq i \leq t} X^{i}$.

(ii) There exist $\mathfrak{g}$-module maps $d_{i}: N_{i} \rightarrow N_{i-1}, 1 \leq i \leq t$, so that

$$
0 \rightarrow N_{t} \stackrel{d_{t}}{\rightarrow} N_{t-1} \stackrel{d_{t-1}}{\rightarrow} \cdots \stackrel{d_{1}}{\rightarrow} N_{0} \stackrel{\epsilon}{\rightarrow} L \rightarrow 0
$$

is complex; i.e., $d_{i} \circ d_{i-1}=0$.

(iii) For all $i$ and $w \in X^{i}$, the restriction of $d_{i}$ to $N_{w \mu-\rho}$ is nonzero.

(iv) For $0 \leq i \leq t, \quad H^{i}\left(\mathfrak{u}^{+}, L\right) \simeq \bigoplus_{w \in X^{i}} F_{w \mu-\rho}$. 
Remarks.

(1) If $L$ is a generalized Kostant module, then the set $X$ and its grading are uniquely determined by (iv).

(2) The Killing form induces a nondegenerate pairing of $S(\mathfrak{u})$ and $S\left(\mathfrak{u}^{-}\right)$. This gives a contragradient pairing of $H^{i}\left(\mathfrak{u}^{+}, L\right)=\operatorname{Ext}_{\mathfrak{u}}^{i}(\mathbb{C}, A)$ and $\operatorname{Tor}_{i}^{\mathfrak{u}^{-}}\left(\mathbb{C}, A^{\vee}\right)$, where $A^{\vee}$ denotes the contragradient dual to $A$. Note that each simple module here is equal to its contragradient dual. Therefore, the identity 2.7(iv) can be interpreted as the corresponding identity for $\operatorname{Tor}_{i}^{\mathfrak{u}^{-}}(\mathbb{C}, L)$.

(3) In [C] the notion of a Kostant module is defined via the homology of the "Iwasawa" Borel subalgebra $\mathfrak{b}^{\sim}$ and its nilradical $\mathfrak{n}^{\sim}$. Following this lead we call $L=L_{\lambda}$ a Kostant module if $\mu$ is regular integral and if the data $X=\bigcup_{0 \leq i \leq t} X^{i}$ is given by $\lambda+\rho=w_{0} \mu$ and $X^{i}=\left\{w \in W^{S} \mid w \prec\right.$ $w_{0}$ and $\left.\ell(w)-\ell\left(w_{0}\right)=i\right\}$.

2.8. Theorem. Suppose $L$ is a generalized Kostant module. Then the complex in 2.7(ii) is a resolution of $L$.

We will prove the theorem in 2.11. The proof is a modification of the argument in Lepowsky [ $\mathrm{L}$ Theorem 4.3] which proves Theorem 2.6 above. We will prove exactness at each term in the complex 2.7 (ii) by induction on the index. Set $\mathfrak{q}^{-}=$ $\mathfrak{p}^{-} \oplus \mathfrak{k}$.

2.9. Lemma [L, Lemma 4.8(ii)]. Let $X$ be a module in the category $\mathcal{O}_{S}$ and $Y$ any $\mathfrak{q}^{-}$-module which is a weight module. Then any $\mathfrak{q}^{-}$-module homomorphism $\varphi: Y \rightarrow X$ is surjective if and only if the induced map $\bar{\varphi}: Y / \mathfrak{u}^{-} Y \rightarrow X / \mathfrak{u}^{-} X$ is surjective.

2.10. Lemma. Suppose $B \subset C$ are $\mathfrak{g}$-modules in the category $\mathcal{O}\left(\mathfrak{g}, \mathfrak{q}_{S}\right)_{\mu}$.

(a) There exist $x_{i} \in W, 1 \leq i \leq t$, and a $\mathfrak{g}$-module filtration of $C$ of the form $C=C_{t} \supset C_{t-1} \supset \cdots \supset C_{1} \supset C_{0}=0$ such that

$$
C / \mathfrak{u}^{-} C \simeq \sum_{1 \leq i \leq t} F\left(\mathfrak{r}, x_{i} \mu-\rho\right)
$$

and $C_{i} / C_{i-1}$ is a quotient of $N\left(x_{i} \mu-\rho\right)$ for all $1 \leq i \leq t$.

(b) Suppose $B \subset \mathfrak{u}^{-} C$ and $L_{\xi}$ is a Jordan-Holder component of $B$. Then there exist $x, y \in D$ such that $\xi=x \mu-\rho, x \neq y, x \prec y$ and $L_{y \mu-\rho}$ is a JordanHolder component of $C$.

Proof. We first prove $(a)$. Choose $x_{1}$ such that $x_{1} \mu-\rho$ is a highest weight of $C$ with highest weight vector $c_{1}$. Set $C_{1}$ (resp. $F_{1}$ ) equal to the $\mathfrak{g}$-module (resp. r-module) generated by $c_{1}$. Then $C_{1}=F_{1} \oplus\left(C_{1} \cap \mathfrak{u}^{-} C\right)$. Let $j \geq 1$ and assume that $C_{j}$ and $F_{j}$ have been defined with vector $c_{j} \in C_{j}$ such that

$$
F_{j}=U(\mathfrak{r}) c_{j}, C_{j}=C_{j-1}+U\left(\mathfrak{u}^{-}\right) F_{j} \quad \text { and } \quad C_{j} /\left(C_{j} \cap \mathfrak{u}^{-} C\right) \simeq \sum_{1 \leq i \leq j} F_{i} .
$$

Now set $c_{j+1}$ equal to any weight vector in $C$ that projects to a highest weight vector in $C / C_{j}$ and generates an irreducible $\mathfrak{r}$-module. Let $F_{j+1}=U(\mathfrak{r}) c_{j+1}$ and let $C_{j+1}$ equal the $\mathfrak{g}$-module generated by $F_{j+1}$ and $C_{j}$. Assertion (a) follows from this.

We now prove (b). Set $L=L_{\xi}$ and choose a submodule $B_{1} \subset B$ with $L$ a submodule of $B / B_{1}$. Now apply part (a) to $A=C / B_{1}$, obtaining the filtration 
$A_{i}$ and the r-modules $F_{i}$. Choose $j$ maximal with $L$ not contained in $A_{j}$. Then $L$ injects into $A_{j+1} / A_{j}$ which is a quotient of $N\left(x_{j+1} \mu-\rho\right)$. Since $L \subset \mathfrak{u}^{-} A$, $x_{j+1} \mu-\rho \neq \xi$ and so $x \neq x_{j+1}$. Setting $y=x_{j+1}$ completes the proof.

2.11. Proof of Theorem 2.8. For any module $A$ we write $\operatorname{Tor}_{i}(A)$ in place of the more precise $\operatorname{Tor}_{i}^{\mathfrak{u}^{-}}(\mathbb{C}, A)$. Then $\operatorname{Tor}_{0}(A) \simeq A / \mathfrak{u}^{-} A$. Set $A$ equal to the kernel of $\epsilon$ and consider the short exact sequences

$$
0 \rightarrow A \rightarrow N_{0} \stackrel{\epsilon}{\rightarrow} L \rightarrow 0 \quad \text { and } \quad 0 \rightarrow K \rightarrow N_{1} \stackrel{d_{1}}{\rightarrow} I \rightarrow 0,
$$

where $I$ equals the image of $N_{1}$ and $K$ is the kernel. Then $I / \mathfrak{u}^{-} I=\operatorname{Tor}_{0}(I)$ is the image of $\operatorname{Tor}_{0}\left(N_{1}\right) \simeq \sum_{x \in X^{1}} F_{x \mu-\rho}$. Using 2.7(iii) and $K \subset \mathfrak{u}^{-} N_{1}, \operatorname{Tor}_{0}(K)$ and $\operatorname{Tor}_{0}\left(N_{1}\right)$ have no $\mathfrak{r}$-modules in common. Thus we have an isomorphism

$$
\operatorname{Tor}_{0}(I) \simeq \operatorname{Tor}_{0}\left(N_{1}\right) \simeq \sum_{x \in X^{1}} F_{x \mu-\rho}
$$

The first of the short exact sequences in $(*)$ induces an exact sequence

$$
0 \rightarrow \operatorname{Tor}_{1}(L) \rightarrow \operatorname{Tor}_{0}(A) \rightarrow \operatorname{Tor}_{0}\left(N_{0}\right) \rightarrow \operatorname{Tor}_{0}(L) \rightarrow 0 .
$$

Since $\operatorname{Tor}_{0}\left(N_{0}\right) \simeq \mathbb{C}_{\lambda}$, this resolution splits into two isomorphisms:

$$
\operatorname{Tor}_{1}(L) \simeq \operatorname{Tor}_{0}(A) \quad \text { and } \quad \mathbb{C}_{\lambda} \simeq \operatorname{Tor}_{0}\left(N_{0}\right) \simeq \operatorname{Tor}_{0}(L) .
$$

This proves that $\operatorname{Tor}_{0}(I)$ and $\operatorname{Tor}_{0}(A)$ are isomorphic.

Now $I \subset A \subset N_{0}$ and $A \subset \mathfrak{u}^{-} N_{0}$. We claim that the inclusion of $I$ in $A$ induces an inclusion $I / \mathfrak{u}^{-} I \rightarrow A / \mathfrak{u}^{-} A$, i.e., $I \cap \mathfrak{u}^{-} A=\mathfrak{u}^{-} I$. Suppose not. Then by Lemma 2.10(a) there exists $x \in X^{1}$ with $F_{x \mu-\rho}$ contained in $\operatorname{Tor}_{0}(I)$ and $L_{x \mu-\rho}$ occurring as a Jordan-Holder component of $I$ which is contained in $\mathfrak{u}^{-} A$. Applying Lemma 2.10(b), we obtain $y \neq x, x \prec y$ with $L_{y \mu-\rho}$ occurring as a Jordan-Holder component of $A$. If $L_{y \mu-\rho}$ is not contained in $\mathfrak{u}^{-} A$, then we have $x \prec y$ with both contained in $X^{1}$. If $L_{y \mu-\rho}$ is contained in $\mathfrak{u}^{-} A$, then repeat the argument to obtain $z \in D$ with $y \prec z$ and $L_{z \mu-\rho}$ occurring as a Jordan Holder component of $A$. Repeat this process until we obtain $w \in D$ with $x \prec w$ and $L_{w \mu-\rho}$ is not contained in $\mathfrak{u}^{-} A$. So $x \prec w$ and both are contained in $X^{1}$. This contradicts 2.7(i) and proves the claim.

We now prove $A=I$. By the previous paragraph the induced map $\operatorname{Tor}_{0}(I) \rightarrow$ $\operatorname{Tor}_{0}(A)$ is injective and hence by (**) is an isomorphism. Then Lemma 2.9 implies that the inclusion of $I$ into $A$ must also be an isomorphism, $A=I$. This proves exactness at $N_{0}$.

We now consider the general case. The argument is similar. Assume $j>0$ and the complex is exact at $N_{i}$ for $0 \leq i \leq j-1$. Set $A$ equal to the kernel of $d_{j}$ and $I$ equal to the image of $d_{j+1}$. We now prove $I=A$. Consider the resolutions

$$
0 \rightarrow A \rightarrow N_{j} \stackrel{d_{j}}{\rightarrow} N_{j-1} \stackrel{d_{j-1}}{\rightarrow} \cdots \stackrel{d_{1}}{\rightarrow} N_{0} \stackrel{\epsilon}{\rightarrow} L \rightarrow 0
$$

and

$$
0 \rightarrow K \rightarrow N_{j+1} \stackrel{d_{j+1}}{\rightarrow} I \rightarrow 0 .
$$

From the set of associated short exact sequences and the freeness of each $N_{i}$, we obtain that $\operatorname{Tor}_{0}(A) \simeq \operatorname{Tor}_{j+1}(L)$. From the second resolution and 2.7(iii) and (iv), the inclusion $K \subset \mathfrak{u}^{-} N_{j+1}$ implies $\operatorname{Tor}_{0}(I) \simeq \operatorname{Tor}_{0}\left(N_{j+1}\right) \simeq \operatorname{Tor}_{j+1}(L)$. This proves $\operatorname{Tor}_{0}(A)$ and $\operatorname{Tor}_{0}(I)$ are isomorphic. 
Next we argue as above that the induced map $I / \mathfrak{u}^{-} I \rightarrow A / \mathfrak{u}^{-} A$ is an injection. Suppose this is not the case. Then by Lemma 2.10(b) we obtain $x, y \in D$ with $F_{x \mu-\rho} \subset \mathfrak{u}^{-} A \cap I$ and not contained in $\mathfrak{u}^{-} I, x \neq y, x \prec y$ and $L_{y \mu-\rho}$ occurring as a Jordan-Holder component of $A$. Then as above we may repeat this process to obtain $w$ with $x \neq w, x \prec w$ and $F_{w \mu-\rho}$ contained in $\operatorname{Tor}_{0}(A)$. Then both $x$ and $w$ lie in $X^{j+1}$ which contradicts 2.7(i). This proves the map $\operatorname{Tor}_{0}(I)$ into $\operatorname{Tor}_{0}(A)$ is injective and since they are isomorphic $\mathfrak{r}$-modules, the map is an isomorphism. Then Lemma 2.9 implies that $I=A$. This completes the induction step and the proof.

\section{Resolutions of Unitary highest Weight MODUleS}

3.1. The reduced Hermitian symmetric pair. In [E] a formula for the $\mathfrak{p}^{+}$ cohomology of unitary highest weight modules is given for Hermitian symmetric pairs of classical type. In [EW] these formulas are used to give a generalized BGG resolution in the classical cases. Here we formulate these results for arbitrary Hermitian symmetric pairs and extend the proof to the exceptional groups. In the language of the previous section, we prove that each unitary highest weight representation is a generalized Kostant module with data $X$. We describe the sets $X$ and their Kostant gradings. Our answer is given in terms of the reduced Hermitian symmetric pair which we now define.

3.2. Definition. Let $\lambda \in \mathfrak{h}^{*}$ and let $\Psi_{\lambda}$ be the set of roots in $\Delta$ that are orthogonal to $\lambda+\rho$. Following $[\mathrm{E}]$ we then define a root system $\Delta_{\lambda}$ as follows. Let $W_{\lambda}$ be the subgoup of $W$ that is generated by the reflections $s_{\beta}$ with $\beta$ satisfying the following conditions:

(i) $\beta \in \Delta_{n}^{+}$and $\left(\lambda+\rho, \beta^{\vee}\right)$ is a positive integer;

(ii) $\beta$ is orthogonal to $\Psi_{\lambda}$;

(iii) $\quad \beta$ is short if $\Psi_{\lambda}$ contains a long root.

Let $\Delta_{\lambda}$ be the subset of roots $\alpha \in \Delta$ with $s_{\alpha} \in W_{\lambda}$. Then $\Delta_{\lambda}$ is an abstract root system and $W_{\lambda}$ is the associated Weyl group. Let $\Delta_{\lambda, c}=\Delta_{c} \cap \Delta_{\lambda}, \Delta_{\lambda}^{+}=$ $\Delta^{+} \cap \Delta_{\lambda}$ and $\Delta_{\lambda, c}^{+}=\Delta_{c}^{+} \cap \Delta_{\lambda}$. Let $\mathfrak{g}_{\lambda}$ and $\mathfrak{k}_{\lambda}$ be the complex Lie algebras with Cartan subalgebra $\mathfrak{h}$ and root systems $\Delta_{\lambda}$ and $\Delta_{\lambda, c}$. So $\mathfrak{g}_{\lambda}=\mathfrak{h} \oplus \sum_{\alpha \in \Delta_{\lambda}} \mathfrak{g}^{\alpha}$ and $\mathfrak{k}_{\lambda}=\mathfrak{h} \oplus \sum_{\alpha \in \Delta_{\lambda, c}} \mathfrak{g}^{\alpha}$. Note that these Lie algebras are the complexified Lie algebras of a Hermitian symmetric pair $\left(G_{\lambda}, K_{\lambda}\right)$. Also note that in general $\mathfrak{g}_{\lambda}$ is not a subalgebra of $\mathfrak{g}$. We call both $\left(G_{\lambda}, K_{\lambda}\right)$ and $\left(\mathfrak{g}_{\lambda}, \mathfrak{k}_{\lambda}\right)$ the reduced Hermitian symmetric pair associated to $\lambda$.

3.3. Put $\rho_{\lambda}=\frac{1}{2} \sum_{\alpha \in \Delta_{\lambda}^{+}} \alpha$ and define $W_{\lambda}^{c}=\left\{w \in W_{\lambda} \mid w \Delta_{\lambda}^{+} \supseteq \Delta_{\lambda, c}^{+}\right\}$. Note that $W_{\lambda, c} \subseteq W_{c}$ but $W_{\lambda}^{c} \nsubseteq W^{c}$ in general. Let $\ell_{\lambda}$ denote the length function on $W_{\lambda}$. Set

$$
W_{\lambda}^{c, i}=\left\{w \in W_{\lambda}^{c} \mid \ell_{\lambda}(w)=i\right\} .
$$

Comparing with 2.2, note that in general $\ell_{\lambda}$ does not equal the restriction of $\ell$ to $W_{\lambda}$. For any $\lambda \in \mathfrak{h}^{*}$ with $\left(\lambda, \alpha^{\vee}\right) \in \mathbb{R}$ for all $\alpha \in \Delta$, let $\lambda^{+}$denote the unique element in the $W_{c^{-}}$-orbit of $\lambda$ which is $\Delta_{c}^{+}$-dominant.

3.4. Now we make the connections with the graded subsets of $D$ which parametrize the BGG resolutions in section two. Recall the double cosets in 2.5. Choose $\mu$ dominant and $w_{0} \in W$ with $\lambda+\rho=w_{0} \mu$. 
Lemma. For $x \in W_{\lambda}^{c}$, the double coset $W_{c} x w_{0} W_{T}$ is of maximal size $\left|W_{c}\right|\left|W_{T}\right|$ and contains a unique element $\delta(x)$ of minimal length.

Proof. Each element of $\Psi_{\lambda}$ is fixed by $x$. Suppose that $(x(\lambda+\rho), \alpha)=0$ for some compact root $\alpha$. Then $x^{-1} \alpha \in \Psi_{\lambda}$ which implies $\alpha \in \Psi_{\lambda}$. This is a contradiction. Hence the double coset has maximal size. An elementary tensoring argument proves the existence of the minimal length representative.

3.5. Recall from 2.5 the subset $D$ of representatives of maximal double cosets in $W_{c} \backslash W / W_{T}$. Then $D$ is a subset of ${ }^{T} W \cap W^{c}$. Define the graded set $X_{\lambda}=\bigcup X_{\lambda}^{i}$ by setting $X_{\lambda}^{i}$ equal to the image of $W_{\lambda}^{c, i}$ under the inclusion

$$
x \rightarrow \delta(x), \quad W_{\lambda}^{c} \hookrightarrow{ }^{T} W \cap W^{c} .
$$

We now turn to the verification that $L$ with data $X$ is a generalized Kostant module. Let $\Delta_{\text {int }}$ denote the integral root system for $\lambda+\rho$. Then $\Delta_{c} \subset \Delta_{\text {int }} \subset \Delta$. Then replacing $\Delta$ by $\Delta_{\text {int }}$ we obtain the length function $\ell_{\text {int }}$ and the Weyl group decomposition $W_{\mathrm{int}} \simeq W_{c} \cdot W_{\mathrm{int}}^{c}$ and the grading $W_{\mathrm{int}}^{c, i}$.

3.6. Lemma [L, Proposition 3.7]. Let $\mu$ be dominant and regular and $\alpha$ a simple root. Suppose $w, w^{\prime} \in W^{c}$ satisfy conditions $w=w^{\prime} s_{\alpha}$ and $\ell_{\mathrm{int}}(w)=\ell_{\mathrm{int}}\left(w^{\prime}\right)+1$. Then the standard map $N(\mathfrak{g}, w \mu-\rho) \rightarrow N\left(\mathfrak{g}, w^{\prime} \mu-\rho\right)$ is nonzero.

Proof. In $[\mathrm{L}$, the proposition is stated only for integral points but the proof given there applies to the more general case as well.

3.7. Theorem [DES] Theorem 4.1]. Suppose that $L_{\lambda}$ is unitary. Then for $i \geq 0$,

$$
H^{i}\left(\mathfrak{p}^{+}, L_{\lambda}\right) \simeq \bigoplus_{w \in W_{\lambda}^{c, i}} F_{\delta(w) \mu} .
$$

3.8. Corollary. Suppose that $L_{\lambda}$ is a unitary highest weight module. Then $L_{\lambda}$ has a resolution of the form

$$
0 \rightarrow N_{p_{\lambda}} \rightarrow \cdots \rightarrow N_{1} \rightarrow N_{0} \rightarrow L_{\lambda} \rightarrow 0
$$

with

$$
N_{i}=\bigoplus_{w \in W_{\lambda}^{c, i}} N_{\delta(w) \mu}
$$

The length $p_{\lambda}$ of the resolution is equal to $\operatorname{dim} \mathfrak{p}_{\lambda}^{+}$.

Proof. Two proofs are sketched in [EW] which cover the classical cases. Here we will complete the result by giving a proof for the exceptional cases. Since the argument of Lepowsky even for the classical cases must be modified at several points and the details are not available elsewhere we begin with some comments on the proofs in the classical cases.

Suppose we are in one of the five classical cases, $L=L_{\lambda}$ and $\lambda+\rho$ is dominant and regular. If in addition $\lambda+\rho$ is integral, then $L$ is finite dimensional and Corollary 3.8 reduces to Theorem 2.6. If $\lambda+\rho$ is dominant and regular but not integral, let $\Delta_{\text {int }}$ denote integral root system. Then we are in one of two cases. Either $\mathfrak{g} \simeq \mathfrak{s o}(2,2 n-1)$ and the category $\mathcal{O}(\mathfrak{g})_{\lambda+\rho}$ is equivalent to the category $\mathcal{O}$ for $\mathfrak{s l}(2)$ or $\mathfrak{g} \simeq \mathfrak{s p}(n)$ and $L$ is one of the two components of the Weil representation. In the first case one can verify Corollary 3.8 directly. So suppose we are in the second case. Here $\mathfrak{g} \simeq \mathfrak{s p}(n), W_{\lambda}$ is the Weyl group for type $D_{n}$ and $\delta(x)=x$ for all $W_{\lambda}^{c}$. 
Using the constants associated with this Weyl group [BGG] and the maps induced by the standard maps we find that $L$ satisfies 2.7(i) and (ii). Then Lemma 3.6 implies 2.7(iii) and Theorem 3.7 gives 2.7(iv) and so the corollary now follows from Theorem 2.8. This is the first application of what we referred to as the Lepowsky argument.

The next step is a reduction by equivalence of category following the arguments in $\left[\mathrm{E}\right.$. Suppose $\lambda+\rho$ is not regular dominant. Choose a $\Delta^{+}$-dominant $\mu$ with $\lambda+\rho=w_{0} \mu$. Then there is an equivalence of category $T_{\lambda}$ defined from the category $\mathcal{C}=\mathcal{O}_{S \cap \Delta_{\lambda}}\left(\mathfrak{g}_{\lambda}\right)_{\lambda+\rho}$ to a subcategory $\mathcal{C}^{\prime} \subset \mathcal{O}_{S}(\mathfrak{g})_{\lambda+\rho}$. This equivalence is defined case by case in $[\mathrm{E}]$ and has the essential property, for all $x \in W_{\lambda}^{c}$,

$$
T_{\lambda}: N\left(\mathfrak{g}_{\lambda}, x(\lambda+\rho)-\rho_{\lambda}\right) \longmapsto N(\mathfrak{g}, \delta(x) \mu-\rho) .
$$

Since $\lambda+\rho$ is dominant regular for $\Delta_{\lambda}^{+}$, we may apply the cases above to obtain the resolution for $L\left(\mathfrak{g}_{\lambda}, \lambda\right)$. Applying the equivalence we obtain Corollary 3.8 for $L$.

Now we turn to the exceptional groups $E_{6}$ and $E_{7}$. Here all the reduction points are integral. So first assume $\lambda+\rho$ is regular integral. From [C, Figures 2 and 3], $\lambda+\rho=x \mu$ where $x$ is one of the vertices $\left\{w_{0}, w_{1}, w_{2}, w_{3}, w_{4}, w_{5}, w_{7}, w_{12},, w_{26}\right\}$ for $E_{6}$ and vertices $\left\{w_{0}, w_{1}, w_{2}, w_{3}, w_{4}, w_{5}, w_{6}, w_{8}, w_{16}, w_{55}\right\}$ for $E_{7}$. The last vertex corresponds to the trivial representation in each case. There the corollary follows from Theorem 2.6. So we assume $x \neq w_{26}$ or $w_{55}$.

3.9. Lemma. Suppose $\mathfrak{g}$ is of type $E_{6}$ or $E_{7}, L_{\lambda}$ is unitary and $\lambda+\rho$ is regular. Then the map $x \rightarrow \delta(x)$ is an order preserving injection from the poset $W_{\lambda}^{c}$ onto its image $D^{\prime} \subset W^{c}$. Moreover, its inverse is also order preserving. The map is an isomorphism of the Bruhat order on $W_{\lambda}^{c}$ onto the restriction to $D^{\prime}$ of the Bruhat order on $W^{c}$.

Proof. This is a straightforward verification. For details see [C].

Combining this lemma and Lemma 3.6, we find that the data $X_{\lambda}^{i}$ as defined in 3.5 is a Kostant grading which satisfies 2.7(i), (ii) and (iii). Then Theorem 3.7 implies 2.7(iv) and so $L$ is a generalized Kostant module. Theorem 2.8 now finishes the proof of Corollary 3.8 for regular points.

Now we turn to the singular points. We begin with the $E_{6}$ case. Recall BGG reciprocity [ES1, Proposition 2.2]. Let $P_{\nu}$ be the projective cover of the simple module $L_{\nu}$. Then $P_{\nu}$ has a generalized Verma flag and the multiplicity of $N_{\nu}$ in a generalized Verma flag of $P_{\xi}$ is equal to the Jordan-Holder multiplicity of $L_{\xi}$ in $N_{\nu}$. Then [ES2, Proposition 2.3] gives the Verma flag multiplicities. In turn this gives with BGG reciprocity the Jordan-Holder series of each generalized Verma module. Identify the semi-regular highest weights plus rho with the linearly ordered set $v_{0}, v_{1}, v_{2}, v_{3}, v_{4}, v_{5}$ and let $N_{v_{i}}\left(\right.$ resp. $\left.L_{v_{i}}\right)$ denote the module $N\left(\mathfrak{g}, v_{i}(\lambda+\rho)-\rho\right)$ (resp. $L\left(\mathfrak{g}, v_{i}(\lambda+\rho)-\rho\right)$ ). We find $N_{v_{0}}$ is simple and for $1 \leq i \leq 5, N_{v_{i}}$ has JordanHolder components $L_{v_{i}}$ and $L_{v_{i-1}}$. Moreover, the latter is the socle. From this we can directly construct the generalized BGG resolution for any of the vertices in this case. All singular reduction points are of this form. Therefore, the corollary holds for $E_{6}$.

For $E_{7}$ the singular reduction points are either semi-regular or have an $A_{1} \times A_{1}$ singularity. By [ES2, Lemma 3.5] the category for an $A_{1} \times A_{1}$ singularity looks like the $\mathfrak{s l}(2)$ category with two simple modules. Both have the desired cohomology formulas and we see directly that both have generalized BGG resolutions. For 
the semi-regular points, ES2, Proposition 3.8] determines the generalized Verma module flags of the projective covers. In turn with BGG reciprocity we can compute the Jordan-Holder series for each generalized Verma module. The semi-regular category corresponds to the following diagram:

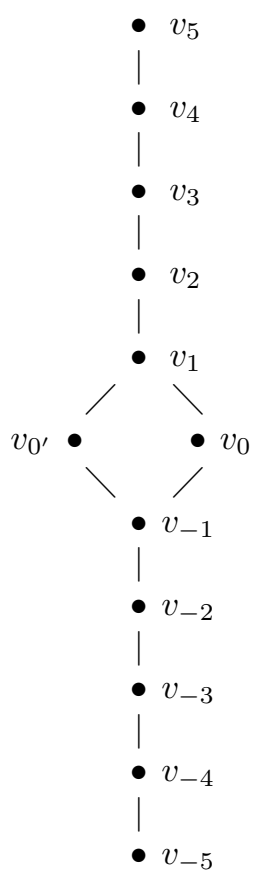

The Jordan-Holder components and socles of generalized Verma modules in this category are given in the following table:

$\begin{array}{cll}\begin{array}{c}\text { generalized } \\ \text { Verma module }\end{array} & \text { Jordan-Holder components } & \text { socle } \\ N_{5} & L_{5}, L_{4}, L_{-5} & \\ N_{4} & L_{4}, L_{3}, L_{-4}, L_{-5} & L_{-4} \\ N_{3} & L_{3}, L_{2}, L_{-3}, L_{-4} & L_{-3} \\ N_{2} & L_{2}, L_{1}, L_{-2}, L_{-3} & L_{-2} \\ N_{1} & L_{1}, L_{0}, L_{0^{\prime}}, L_{-1}, L_{-2} & L_{-1} \\ N_{0} & L_{0}, L_{-1} & L_{-1} \\ N_{0^{\prime}} & L_{0^{\prime}}, L_{-1} & L_{-1} \\ N_{-1} & L_{-1}, L_{-2} & L_{-2} \\ N_{-2} & L_{-2}, L_{-3} & L_{-3} \\ N_{-3} & L_{-3}, L_{-4} & L_{-4} \\ N_{-4} & L_{-4}, L_{-5} & L_{-5} \\ N_{-5} & L_{-5} & L_{-5}\end{array}$

By construction (cf. [ES2]) the projective covers $P_{v_{-5}}, P_{v_{-4}}, P_{v_{-3}}, P_{v_{-2}}$ and $P_{v_{-1}}$ are self dual. This implies that Socle $N_{j}=$ Socle $P_{-j}=L_{-j}$ for $1 \leq j \leq 5$. From the Jordan-Holder table we obtain the other socle information. This is listed in the far right column. Directly, as a result of these tables, if $\lambda+\rho$ corresponds to one of the vertices $\left\{v_{-5}, v_{-4}, v_{-3}, v_{-2}, v_{-1}, v_{0}, v_{0^{\prime}}, v_{5}\right\}$, then we can define maps $d_{j}$ so that 
2.7(i)-(iii) all hold. All semiregular $\lambda+\rho$ for $E_{7}$ with $L_{\lambda}$ unitary correspond to these vertices. Then using Theorem 2.8 and Theorem 3.7 we obtain Corollary 3.8 for $E_{7}$. This completes the entire proof.

3.10. Corollary to Proof. Suppose $\mathfrak{g}$ is of type $E_{6}$ and $\lambda+\rho$ is singular integral. Then $L_{\lambda}$ is a generalized Kostant module.

\section{The CONE DECOMPosition AND Unitary StRata}

4.1. The classification of unitary highest weight modules. We review some of the notation from the paper [EHW] on the classification of unitary highest weight modules and the cone decomposition. Let $\beta$ denote the unique maximal root in $\Delta^{+}$ and let $\zeta$ be the unique fundamental $\operatorname{root}\left(\zeta, \beta^{\vee}\right)=1$. Let $\lambda_{0}$ be a $\Delta_{c}^{+}$-dominant integral element of $\mathfrak{h}^{*}$ such that $\left(\lambda_{0}+\rho, \beta^{\vee}\right)=0$. If $L_{\lambda}$ is unitary, then there exists a unique $\lambda_{0}$ as above such that $\lambda=\lambda_{0}+z \zeta$ with $z \in \mathbb{R}$. For fixed $\lambda_{0}$ the set of all $z \in \mathbb{R}$ such that the irreducible highest weight module $L_{\lambda_{0}+z \zeta}$ is unitary is as follows:

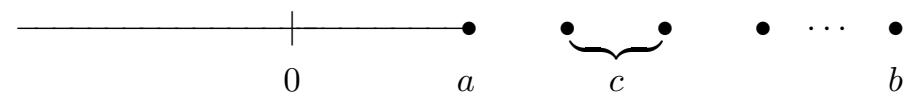

The constants $a$ and $b$ depend on $\lambda_{0}$, whereas the constant $c$ depends only on the Hermitian symmetric pair.

Since in this article we consider resolutions and Hilbert series of unitary highest weight modules $L_{\lambda}$, we are interested in those highest weights $\lambda$ for which $N_{\lambda}$ is reducible (and hence $L_{\lambda}$ has a nontrivial resolution). We call the set of such highest weights the set of reduction points. In the picture above, the reduction points correspond to the equally spaced points between $a$ and $b$. The highest weight corresponding to $z=a$ is called the first reduction point; the highest weight corresponding to $z=b$ is called the last reduction point.

For simplicity, in what follows we only consider Hermitian symmetric pairs with simply laced Dynkin diagram, i.e., root systems of $A D E$-type. In this case the constants $a$ and $b$ mentioned above have some simple interpretations in terms of a certain root sytem, $Q_{\lambda_{0}}$, attached to the line $\lambda_{0}+\mathbb{R} \zeta$.

4.2. The root system $Q_{\lambda_{0}}$. We begin with a general observation on Dynkin diagrams of Hermitian symmetric pairs (which is also valid in the nonsimply laced cases). Consider the extended Dynkin diagram that is obtained from the Dynkin diagram of $\Delta$ by adding an extra node corresponding to $-\beta$ that is connected to the other nodes using the usual rules. For example, if $\Delta$ is the Dynkin diagram of $E_{6}$, then the extended diagram is:

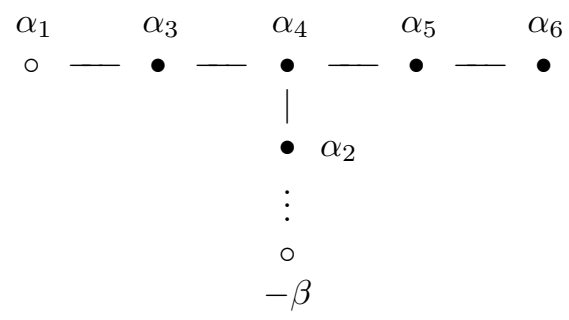

Now delete the node corresponding to the unique simple noncompact root. Then the resulting diagram is again the Dynkin diagram of the root system of a Hermitian 
symmetric pair of the same type with $-\beta$ being the node corresponding to the simple noncompact root. For example, in the case of $E_{6}$ :

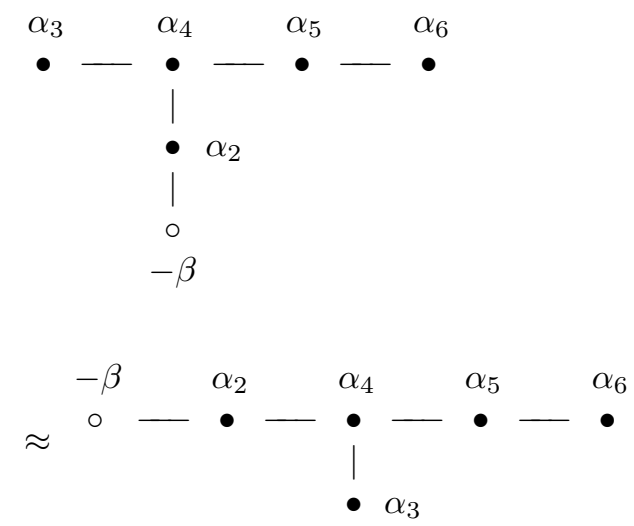

As in [EHW], we attach to $\lambda$ a root system, $Q_{\lambda}$, as follows: the diagram of $Q_{\lambda}$ is the maximal connected subdiagram of the diagram above containing $-\beta$ and having the property that every compact simple root is orthogonal to $\lambda$. For example, consider the case of $E_{6}$ and $\lambda=\omega_{5}-9 \omega_{1}$. To find $Q_{\lambda}$ we first label the nodes corresponding the simple compact roots by the dot products $\left(\lambda, \alpha_{i}^{\vee}\right)$ :

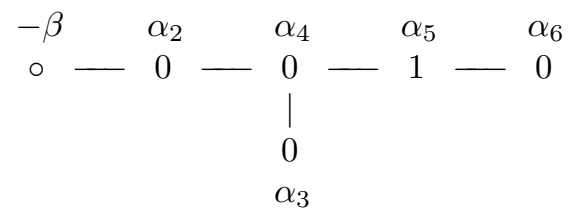

Thus $Q_{\lambda}$ is of type $S U(1,4)$ :

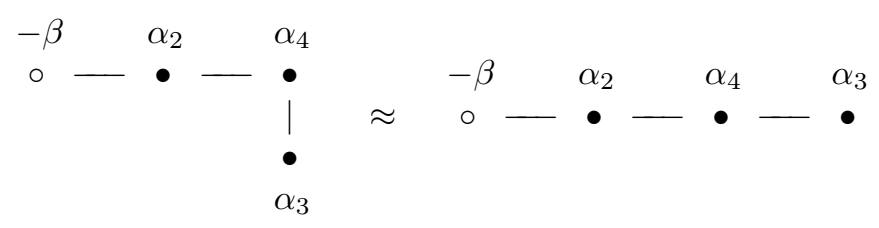

Note that if $\lambda=\lambda_{0}+z \zeta$ as in 4.1 , then $Q_{\lambda}=Q_{\lambda_{0}}$ since $\zeta$ is orthogonal to all compact roots.

The constants $a$ and $b$ from 4.1 (in the simply laced cases) can be expressed in terms of $Q=Q_{\lambda_{0}}$ as follows. Let $r$ be the split rank of $Q$. Then

$$
b=2\left(\rho_{Q, c}, \beta^{\vee}\right)+1 \quad \text { and } \quad a=b-(r-1) c
$$

Here $\rho_{Q, c}$ denotes half the sum of all the positive compact roots of $Q$.

4.3. The cone decomposition. The set of all reduction points can be written as a (disjoint) union of certain translated integral cones. This cone decomposition was explicitly introduced in [DES]. Here, by a translated integral cone in $\mathfrak{h}^{*}$ with vertex $\nu$, we mean a subset of $\mathfrak{h}^{*}$ of the form $\mathcal{C}=\left\{\nu+\sum_{j=1}^{m} a_{j} \pi_{j} \mid a_{j} \in \mathbb{Z}_{+}\right\}$, where $\nu$ and $\pi_{j}$ are $\Delta_{c}$-integral. 
Let $Q$ be a root system of the form $Q=Q_{\lambda}$ for some reduction point $\lambda=\lambda_{0}+z \zeta$. Fixing the root system $Q$ and value $z$ we define a subset $\mathcal{C}_{Q, z}$ of the set of all reduction points by

$$
\mathcal{C}_{Q, z}=\left\{\lambda=\lambda_{0}+z \zeta \mid \lambda \text { is a reduction point and } Q_{\lambda}=Q\right\} .
$$

It was observed in DES that the set $\mathcal{C}_{Q, z}$ is a translated integral cone. More precisely, $\mathcal{C}_{Q, z}=\left\{\nu+\sum_{j=1}^{m} a_{j} \pi_{j} \mid a_{j} \in \mathbb{Z}_{+}\right\}$, where the vertex $\nu$ and the generators $\pi_{j}$ are given as follows. Let $\omega_{i}$ denote the fundamental weight corresponding to the simple root $\alpha_{i}$. Then $\nu=\sum_{i} c_{i} \omega_{i}$ is such that $\left(\nu+\rho, \beta^{\vee}\right)=z$ and the coefficients $c_{i}=\left(\nu, \alpha_{i}^{\vee}\right)$ for compact $\alpha_{i}$ are equal to 1 if $\alpha_{i}$ does not belong to $Q$ but the corresponding node in the extended Dynkin diagram is connected to some node of the diagram of $Q$ and is equal to 0 otherwise. The generators $\pi_{j}$ are the weights of the form $\omega_{i}-\left(\omega_{i}, \beta^{\vee}\right) \zeta$, where $\alpha_{i}$ is a compact root that does not belong to $Q$.

4.4. A finer decomposition of the cones. Let $\Sigma$ denote the set of simple roots in $\Delta^{+}$and for $\alpha \in \Sigma$ let $H_{\alpha}$ denote the hyperplane in $\mathfrak{h}_{\mathbb{R}}^{*}$ that is perpendicular to $\alpha$. (Here we identify $\mathfrak{h}^{*}$ with $\mathfrak{h}$ via the Killing form.) For a subset $T \subseteq \Sigma$ define

$$
H_{T}=\bigcap_{\alpha \in T} H_{\alpha}
$$

Let $\mathfrak{C}_{T}^{+}$denote the interior in $H_{T}$ of the intersection $H_{T} \cap \overline{\mathfrak{C}^{+}}$. We then can write $\mathfrak{h}_{\mathbb{R}}^{*}$ as a disjoint union as follows:

$$
\mathfrak{h}_{\mathbb{R}}^{*}=\bigcup_{T \subseteq \Sigma} \bigcup_{w \in{ }^{T} W} w \mathfrak{C}_{T}^{+}
$$

The following lemma can be verified by a case by case analysis.

Lemma. Suppose the set $\left\{\lambda \in \mathcal{C}_{Q, z} \mid \lambda+\rho \in w \mathfrak{C}_{T}^{+}\right\}$is nonempty. Then $\left\{\lambda \in \mathcal{C}_{Q, z} \mid\right.$ $\left.\lambda+\rho \in w \mathfrak{C}_{T}^{+}\right\}$is itself a translated integral cone with a unique vertex.

Definition. We will call $\left\{\lambda \in \mathcal{C}_{Q, z} \mid \lambda+\rho \in w \mathfrak{C}_{T}^{+}\right\}$, provided it is nonempty, the unitary stratum with parameters $(w, T)$. Its vertex is denoted $\nu_{w, T}$. At the end of this section we will explicitly list all the unitary strata for the exceptional groups.

4.5. Posets for $E_{6}$ and $E_{7}$. Many of the calculations to come later are direct consequences of the poset structure of $W^{c}$. The partially ordered set $W^{c}$ is given 
by the following figures for $E_{6}$ and $E_{7}$ respectively:

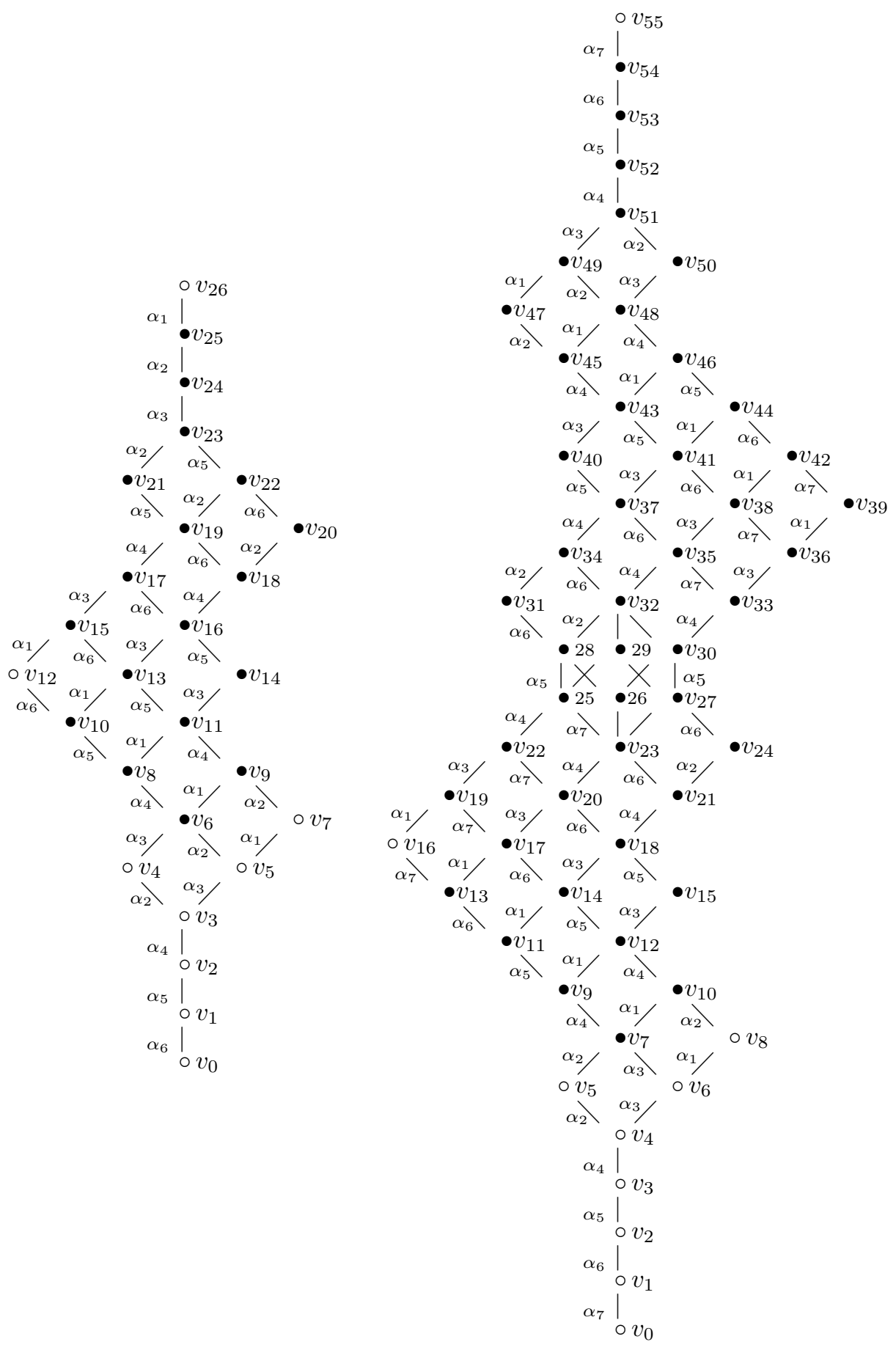


4.6. Root orderings for $\Delta_{n}^{+}$of $E_{6}$ and $E_{7}$. The positive noncompact roots and their Dynkin diagrams are given as follows.

Simple roots and extended Dynkin diagram for $E_{6}$ :

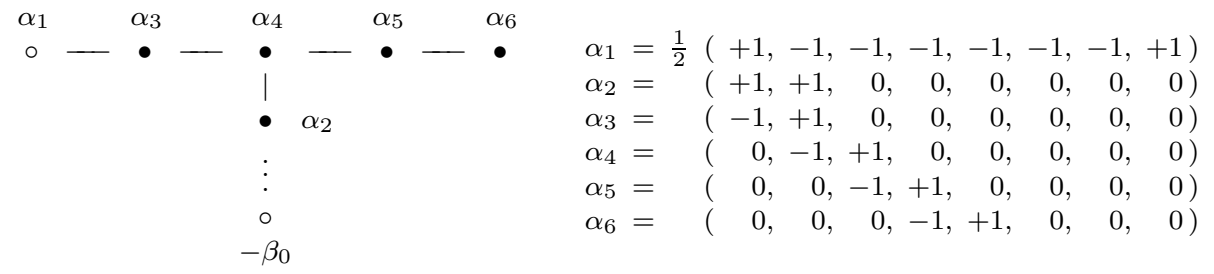

Noncompact positive roots for $E_{6}$ :

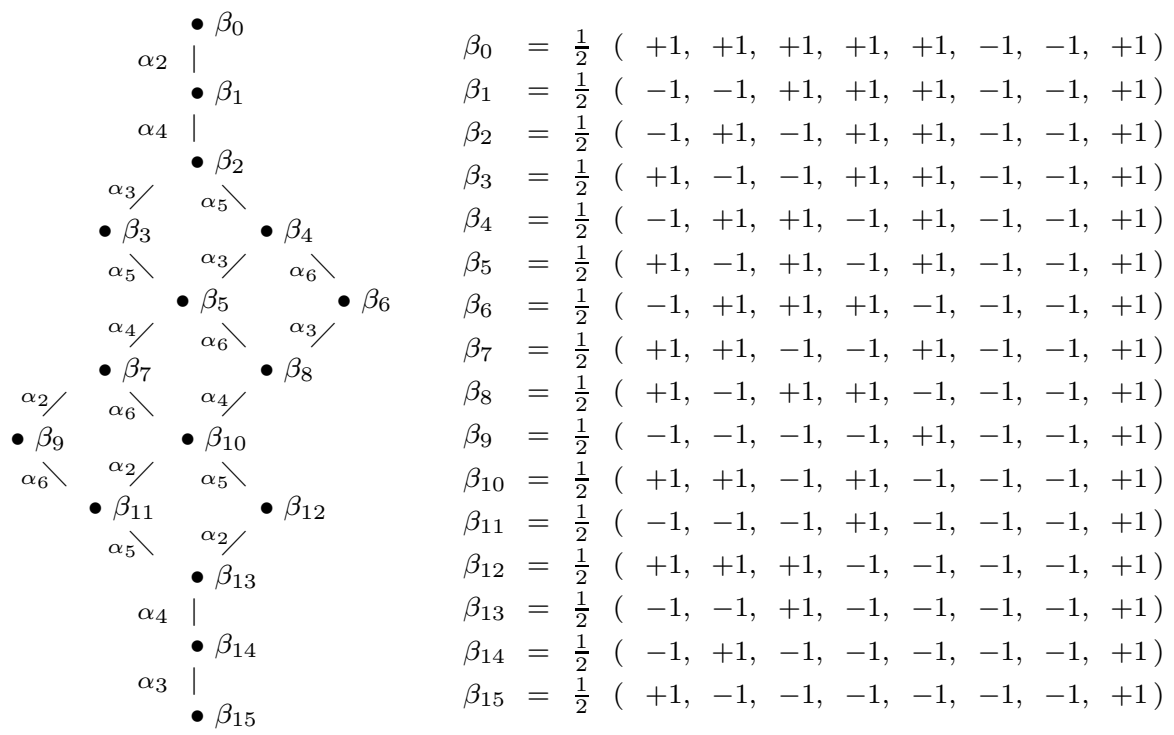

Remark. Here two roots $\beta_{a}$ above $\beta_{b}$ are connected by an edge $\alpha$ if $\beta_{a}=\beta_{b}+\alpha$ with $\alpha$ a simple compact root.

Simple roots and extended Dynkin diagram for $E_{7}$ :

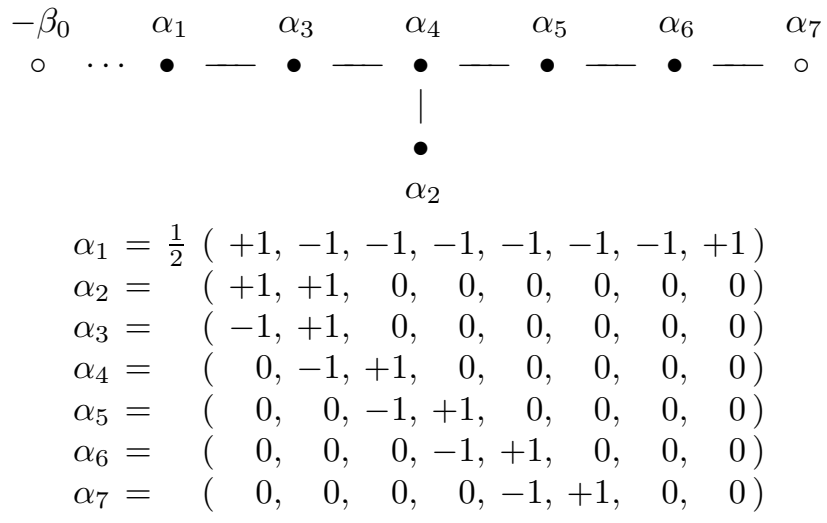


Noncompact positive roots for $E_{7}$ :

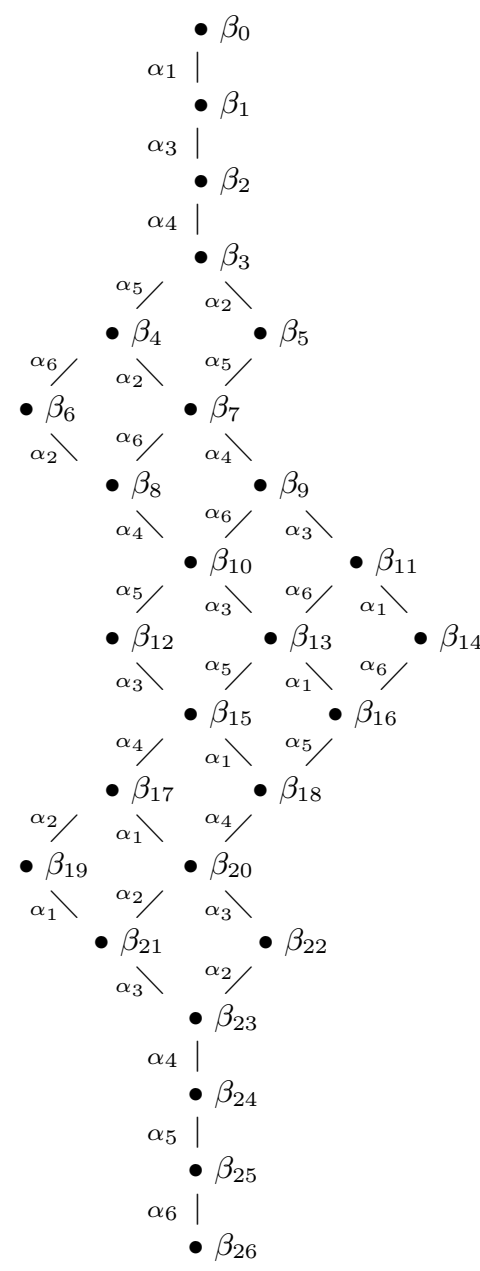

$$
\begin{aligned}
& \beta_{0}=\left(\begin{array}{llllllll}
0, & 0, & 0, & 0, & 0, & 0, & -1, & 1
\end{array}\right) \\
& \beta_{1}=\frac{1}{2}(-1, \quad 1, \quad 1, \quad 1, \quad 1, \quad 1,-1, \quad 1) \\
& \beta_{2}=\frac{1}{2}(1,-1, \quad 1, \quad 1, \quad 1, \quad 1,-1,1) \\
& \beta_{3}=\frac{1}{2}(1, \quad 1,-1, \quad 1, \quad 1, \quad 1,-1,1) \\
& \beta_{4}=\frac{1}{2}(1, \quad 1, \quad 1,-1, \quad 1, \quad 1,-1,1) \\
& \beta_{5}=\frac{1}{2}(-1,-1,-1, \quad 1, \quad 1,1,-1, \quad 1) \\
& \beta_{6}=\frac{1}{2}(1, \quad 1, \quad 1, \quad 1,-1,1,-1, \quad 1) \\
& \beta_{7}=\frac{1}{2}(-1,-1, \quad 1,-1, \quad 1, \quad 1,-1,1) \\
& \beta_{8}=\frac{1}{2}(-1,-1, \quad 1, \quad 1,-1, \quad 1,-1, \quad 1) \\
& \beta_{9}=\frac{1}{2}(-1, \quad 1,-1,-1, \quad 1, \quad 1,-1,1) \\
& \beta_{10}=\frac{1}{2}(-1, \quad 1,-1, \quad 1,-1, \quad 1,-1,1) \\
& \beta_{11}=\frac{1}{2}(1,-1,-1,-1, \quad 1,1,-1, \quad 1) \\
& \beta_{12}=\frac{1}{2}(-1, \quad 1, \quad 1,-1,-1, \quad 1,-1, \quad 1) \\
& \beta_{13}=\frac{1}{2}(1,-1,-1, \quad 1,-1, \quad 1,-1,1) \\
& \beta_{14}=\left(\begin{array}{llllllll}
0, & 0, & 0, & 0, & 1, & 1, & 0, & 0
\end{array}\right) \\
& \beta_{15}=\frac{1}{2}(1,-1, \quad 1,-1,-1, \quad 1,-1,1) \\
& \beta_{16}=\left(\begin{array}{llllllll}
0, & 0, & 0, & 1, & 0, & 1, & 0, & 0
\end{array}\right) \\
& \beta_{17}=\frac{1}{2}(1, \quad 1,-1,-1,-1,1,-1, \quad 1)
\end{aligned}
$$

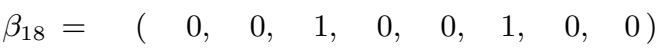

$$
\begin{aligned}
& \beta_{19}=\frac{1}{2}(-1,-1,-1,-1,-1,1,-1, \quad 1) \\
& \beta_{20}=\left(\begin{array}{llllllll}
0, & 1, & 0, & 0, & 0, & 1, & 0, & 0
\end{array}\right) \\
& \beta_{21}=\left(\begin{array}{llllllll}
-1, & 0, & 0, & 0, & 0, & 1, & 0, & 0
\end{array}\right) \\
& \beta_{22}=\left(\begin{array}{llllllll}
1, & 0, & 0, & 0, & 0, & 1, & 0, & 0
\end{array}\right) \\
& \beta_{23}=(0,-1, \quad 0, \quad 0, \quad 0, \quad 1, \quad 0, \quad 0)
\end{aligned}
$$

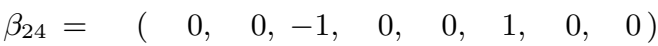

$$
\begin{aligned}
& \beta_{25}=\left(\begin{array}{lllllll}
0, & 0, & 0,-1, & 0, & 1, & 0, & 0
\end{array}\right)
\end{aligned}
$$

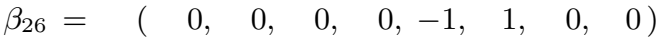

4.7. Example of decomposition into strata. Consider the case of $E_{7}$ when $Q=S O(2,10)$ and $z=9$. This is the most difficult case. In this case the points in the cone $\mathcal{C}_{Q, z}$ are given by $\lambda=a \omega_{6}+(-2 a-8) \omega_{7}$ with $a \geq 1$. We now evaluate $\left(\lambda+\rho, \beta^{\vee}\right)$ for all $\beta \in \Delta_{n}^{+}$. We display these values in a diagram as follows. Start with the poset diagram of $\Delta_{n}^{+}$. Then label each node of the diagram with the corresponding number $\left(\lambda+\rho, \beta^{\vee}\right)$. We are only interested in the nonnegative values and so we ignore the part of the diagram for which the values $\left(\lambda+\rho, \beta^{\vee}\right)$ are negative. What we obtain is a diagram as follows: 


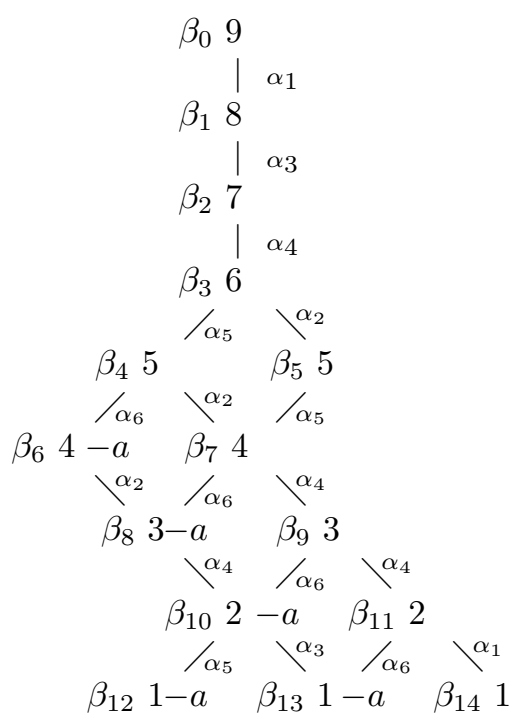

Our calculations will rely on the following lemma.

Lemma. The following are equivalent:

(a) $\lambda+\rho$ lies in the closed chamber $w \overline{\mathfrak{C}^{+}}$;

(b) $\left(\lambda+\rho, w \beta^{\vee}\right) \geq 0$ for all $\beta \in \Delta_{n}^{+}$;

(c) $w \Delta_{n}^{+} \cap \Delta_{n}^{+}=\left\{\beta \in \Delta_{n}^{+} \mid\left(\lambda+\rho, \beta^{\vee}\right) \geq 0\right\}$.

We obtain five different cases depending on the value of $a$, namely $a=1,2$, 3,4 and $\geq 5$. For $a \geq 5$, the parameter $\lambda+\rho$ is integral regular and lies in the chamber $w \mathfrak{C}^{+}$, where $w$ is the unique element of $W^{c}$ with $w \Delta_{n}^{+} \cap \Delta_{n}^{+}=$ $\left\{\beta_{0}, \beta_{1}, \beta_{2}, \beta_{3}, \beta_{4}, \beta_{5}, \beta_{7}, \beta_{9}, \beta_{11}, \beta_{14}\right\}=\Theta$. From the figure in 4.5 we verify that $w=v_{16}$. Thus the parameters of the stratum $\left\{a \omega_{6}+(-2 a-8) \omega_{7} \mid a \geq 5\right\}$ are $(w, T)=\left(v_{16}, \varnothing\right)$. Next consider $a=4$. Then $w \in W^{c}$ is the unique element with $w \Delta_{n}^{+} \cap \Delta_{n}^{+}=\Theta \cup\left\{\beta_{6}\right\}$, which implies by the figure in 4.5 that $w=v_{19}$. One also checks that $\beta_{6}=v_{16} \alpha_{1}$ and hence the parameters of the stratum $\left\{4 \omega_{6}-16 \omega_{7}\right\}$ are $(w, T)=\left(v_{19},\left\{\alpha_{1}\right\}\right)$. Similarly, for $a=3$ and 2 we obtain parameters $(w, T)=\left(v_{22},\left\{\alpha_{3}\right\}\right)$ and $\left(v_{22},\left\{\alpha_{4}\right\}\right)$, respectively. Finally, for $a=1$ we have $w \Delta_{n}^{+} \cap \Delta_{n}^{+}=\left\{\beta_{1}, \beta_{2}, \ldots, \beta_{14}\right\}$. Here there are two singular roots: $\beta_{12}$ and $\beta_{13}$. With $w=v_{32}$ we have $\beta_{12}=v_{32} \alpha_{2}, \beta_{13}=v_{32} \alpha_{5}$. Thus the parameters of the stratum $\left\{\omega-10 \omega_{7}\right\}$ are $(w, T)=\left(v_{32},\left\{\alpha_{2}, \alpha_{5}\right\}\right)$.

4.8. Invariance on stratum. Now we determine invariance properties on strata. Suppose $\xi$ and $\nu$ lie in the positive chamber, have integral difference $\xi-\nu$ and equal stabilizers in $W$, i.e., $\{w \in W \mid w \xi=\xi\}=\{w \in W \mid w \nu=\nu\}$. Applying standard techniques of Zuckerman translation we have an equivalence of categories $\Theta: \mathcal{O}_{\xi} \stackrel{\sim}{\longrightarrow}$ $\mathcal{O}_{\nu}$. Suppose $w \in W^{c}$ and assume both $w \xi$ and $w \nu$ are $\Delta_{c}^{+}$-dominant integral and regular. Then $\Theta N_{w \xi} \simeq N_{w \nu}$ and $\Theta L_{w \xi} \simeq L_{w \nu}$. Applying this equivalence to our modules and resolutions we obtain:

Lemma. The Gelfand-Kirilov dimension, the length of a minimal free resolution and the degree of the numerator polynomial in the Hilbert series are constant on each stratum. 
4.9. Table of strata decomposition. The following tables summarize the decomposition of cones into strata. Each line corresponds to a strata. For each stratum we give the vertex of the stratum, the Gelfand-Kirillov dimension of $L_{\lambda}$ with $\lambda+\rho$ in the stratum, the length $p$ of the generalized BGG resolution and the degree $e$ of the numerator polynomial $P(t)$.

Unitary strata for $E_{6}$ :

\begin{tabular}{|c|c|c|c|c|c|c|}
\hline$Q$ & $z$ & $w$ & $T$ & vertex $\nu_{w, T}$ & GKdim & $p$ \\
\hline$S U(1,1)$ & 1 & $v_{1}$ & $\varnothing$ & $\omega_{2}-12 \omega_{1}$ & 16 & 1 \\
\hline$S U(1,2)$ & 2 & $v_{2}$ & $\varnothing$ & $\omega_{4}-12 \omega_{1}$ & 16 & 2 \\
\hline$S U(1,3)$ & 3 & $v_{3}$ & $\varnothing$ & $\omega_{3}+\omega_{5}-12 \omega_{1}$ & 16 & 3 \\
\hline$S U(1,4)^{(a)}$ & 4 & $\begin{array}{l}v_{4} \\
v_{6}\end{array}$ & $\begin{array}{l}\varnothing \\
\left\{\alpha_{3}\right\}\end{array}$ & $\begin{array}{l}2 \omega_{5}-11 \omega_{1} \\
\omega_{5}-9 \omega_{1}\end{array}$ & $\begin{array}{l}16 \\
16\end{array}$ & $\begin{array}{l}4 \\
1\end{array}$ \\
\hline$S U(1,4)^{(b)}$ & 4 & $\begin{array}{l}v_{5} \\
v_{6}\end{array}$ & $\begin{array}{l}\varnothing \\
\left\{\alpha_{2}\right\}\end{array}$ & $\begin{array}{l}2 \omega_{3}+\omega_{6}-12 \omega_{1} \\
\omega_{3}+\omega_{6}-10 \omega_{1}\end{array}$ & $\begin{array}{l}16 \\
16\end{array}$ & $\begin{array}{l}4 \\
1\end{array}$ \\
\hline$S U(1,5)$ & 5 & $\begin{array}{l}v_{7} \\
v_{9} \\
v_{11}\end{array}$ & $\begin{array}{l}\varnothing \\
\left\{\alpha_{2}\right\} \\
\left\{\alpha_{4}\right\}\end{array}$ & $\begin{array}{l}3 \omega_{3}-12 \omega_{1} \\
2 \omega_{3}-10 \omega_{1} \\
\omega_{3}-8 \omega_{1}\end{array}$ & $\begin{array}{l}16 \\
16 \\
16\end{array}$ & $\begin{array}{l}5 \\
2 \\
2\end{array}$ \\
\hline$S O(2,8)$ & $\begin{array}{l}4 \\
7\end{array}$ & $\begin{array}{l}v_{8} \\
v_{12} \\
v_{15} \\
v_{17} \\
v_{19}\end{array}$ & $\begin{array}{l}\left\{\alpha_{4}\right\} \\
\varnothing \\
\left\{\alpha_{1}\right\} \\
\left\{\alpha_{3}\right\} \\
\left\{\alpha_{4}\right\}\end{array}$ & $\begin{array}{l}\omega_{6}-8 \omega_{1} \\
4 \omega_{6}-8 \omega_{1} \\
3 \omega_{6}-7 \omega_{1} \\
2 \omega_{6}-6 \omega_{1} \\
\omega_{6}-5 \omega_{1}\end{array}$ & $\begin{array}{l}16 \\
16 \\
16 \\
16 \\
16\end{array}$ & $\begin{array}{l}1 \\
\\
8 \\
4 \\
4 \\
4\end{array}$ \\
\hline$E_{6}$ & $\begin{array}{l}8 \\
11\end{array}$ & $\begin{array}{l}v_{24} \\
v_{26}\end{array}$ & $\begin{array}{l}\left\{\alpha_{4}\right\} \\
\varnothing\end{array}$ & $\begin{array}{l}-3 \omega_{1} \\
0\end{array}$ & $\begin{array}{l}11 \\
1\end{array}$ & $\begin{array}{c}5 \\
16\end{array}$ \\
\hline
\end{tabular}

The two cases with $Q=S U(1,4)$ correspond to the following Dynkin diagrams:

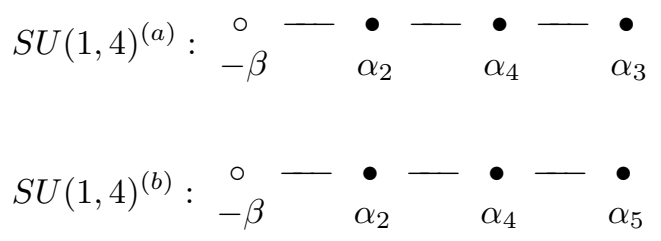


UNITARY HIGHEST WEIGHT MODULES

Unitary strata for $E_{7}$ :

\begin{tabular}{|c|c|c|c|c|c|c|}
\hline$Q$ & $z$ & $w$ & $T$ & vertex $\nu_{w, T}$ & GKdim & $p$ \\
\hline$S U(1,1)$ & 1 & $v_{1}$ & $\varnothing$ & $\omega_{1}-18 \omega_{7}$ & 27 & 1 \\
\hline$S U(1,2)$ & 2 & $v_{2}$ & $\varnothing$ & $\omega_{3}-18 \omega_{7}$ & 27 & 2 \\
\hline$S U(1,3)$ & 3 & $v_{3}$ & $\varnothing$ & $\omega_{4}-18 \omega_{7}$ & 27 & 3 \\
\hline$S U(1,4)$ & 4 & $v_{4}$ & $\varnothing$ & $\omega_{2}+\omega_{5}-18 \omega_{7}$ & 27 & 4 \\
\hline \multirow[t]{2}{*}{$S U(1,5)^{(a)}$} & 5 & $v_{5}$ & $\varnothing$ & $2 \omega_{5}-18 \omega_{7}$ & 27 & 5 \\
\hline & & $v_{7}$ & $\left\{\alpha_{3}\right\}$ & $\omega_{5}-15 \omega_{7}$ & 27 & 1 \\
\hline \multirow[t]{2}{*}{$S U(1,5)^{(b)}$} & 5 & $v_{6}$ & $\varnothing$ & $2 \omega_{2}+\omega_{6}-18 \omega_{7}$ & 27 & 5 \\
\hline & & $v_{7}$ & $\left\{\alpha_{2}\right\}$ & $\omega_{2}+\omega_{6}-16 \omega_{7}$ & 27 & 1 \\
\hline \multirow[t]{3}{*}{$S U(1,6)$} & 6 & $v_{8}$ & $\varnothing$ & $3 \omega_{2}-17 \omega_{7}$ & 27 & 6 \\
\hline & & $v_{10}$ & $\left\{\alpha_{2}\right\}$ & $2 \omega_{2}-15 \omega_{7}$ & 27 & 2 \\
\hline & & $v_{12}$ & $\left\{\alpha_{4}\right\}$ & $\omega_{2}-13 \omega_{7}$ & 27 & 2 \\
\hline \multirow[t]{6}{*}{$S O(2,10)$} & 5 & $v_{9}$ & $\left\{\alpha_{4}\right\}$ & $\omega_{6}-14 \omega_{7}$ & 27 & 1 \\
\hline & 9 & $v_{16}$ & $\varnothing$ & $5 \omega_{6}-18 \omega_{7}$ & 26 & 10 \\
\hline & & $v_{19}$ & $\left\{\alpha_{1}\right\}$ & $4 \omega_{6}-16 \omega_{7}$ & 26 & 5 \\
\hline & & $v_{22}$ & $\left\{\alpha_{3}\right\}$ & $3 \omega_{6}-14 \omega_{7}$ & 26 & 5 \\
\hline & & $v_{25}$ & $\left\{\alpha_{4}\right\}$ & $2 \omega_{6}-12 \omega_{7}$ & 26 & 5 \\
\hline & & $v_{32}$ & $\left\{\alpha_{2}, \alpha_{5}\right\}$ & $\omega_{6}-10 \omega_{7}$ & 26 & 1 \\
\hline \multirow[t]{3}{*}{$E_{7}$} & 9 & $v_{37}$ & $\left\{\alpha_{4}, \alpha_{6}\right\}$ & $-8 \omega_{7}$ & 26 & 1 \\
\hline & 13 & $v_{52}$ & $\left\{\alpha_{4}\right\}$ & $-4 \omega_{7}$ & 17 & 10 \\
\hline & 17 & $v_{55}$ & $\varnothing$ & 0 & 1 & 27 \\
\hline
\end{tabular}

The two cases with $Q=S U(1,5)$ correspond to the following Dynkin diagrams:

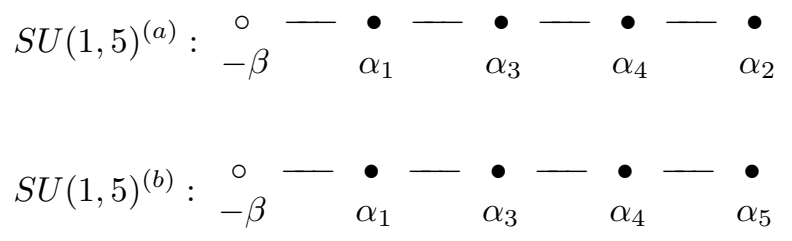

\section{Computing Reduced Root Systems}

5.1. In sections 6 and 7 we give formulas for the generalized BGG resolutions, the Hilbert series, Bernstein degree, etc. of the unitary highest weight modules of the exceptional groups of $E_{6}$ and $E_{7}$, respectively. In order to find the explicit 
resolution of the unitary highest weight module $L_{\lambda}$ with highest weight $\lambda$ one has to determine the reduced root system $\Delta_{\lambda}$, which was introduced in [E]. In the following we describe an algorithm to find $\Delta_{\lambda}$.

5.2. An algorithm to find $\Delta_{\lambda}$. Begin with the set $\Delta_{n}^{+}$of positive noncompact roots described as an ordered set in 4.6. Given the highest weight $\lambda$ of the unitary irreducible highest weight module we compute the scalar product $\left(\lambda+\rho, \beta^{\vee}\right)$ for all $\beta \in \Delta_{n}^{+}$. The $\beta \in \Delta_{n}^{+}$for which $\left(\lambda+\rho, \beta^{\vee}\right)=0$ are the singular (positive) roots for the parameter $\lambda+\rho$. This gives $\Psi_{\lambda} \cap \Delta^{+}$. We next determine the noncompact positive roots orthogonal to $\Psi_{\lambda}$ and with positive integral inner product with $\lambda+\rho$. The set of all these roots is the set $\Delta_{n, \lambda}^{+}$. It inherits a partial ordering from the partial ordering of $\Delta_{n}^{+}$. The simple roots of the root system $\Delta_{\lambda}$ are the roots that arise as differences of successive elements of $\Delta_{n}^{+}$.

5.3. Two examples of the $\Delta_{\lambda}^{+}$calculation. We again consider the case of $E_{7}$ when $Q=S O(2,10)$ and $z=9$ as in 4.7 . In this case the points in the cone $\mathcal{C}_{Q, z}$ are given by $\lambda=a \omega_{6}+(-2 a-8) \omega_{7}$ with $a \geq 1$. We now evaluate $\left(\lambda+\rho, \beta^{\vee}\right)$ for all $\beta \in \Delta_{n}^{+}$. We display these values in a diagram as follows. Start with the poset diagram of $\Delta_{n}^{+}$. Then label each node of the diagram with the corresponding number $\left(\lambda+\rho, \beta^{\vee}\right)$. We are only interested in the nonnegative values and so we ignore the part of the diagram for which the values $\left(\lambda+\rho, \beta^{\vee}\right)$ are negative. What we obtain is a diagram as follows:

$$
\begin{aligned}
& \beta_{0} 9 \\
& \text { | } \alpha_{1} \\
& \beta_{1} 8 \\
& \text { | } \alpha_{3} \\
& \beta_{2} 7 \\
& \text { | } \alpha_{4} \\
& \beta_{3} 6 \\
& \alpha_{5} \quad \alpha_{2} \\
& \beta_{4} 5 \quad \beta_{5} 5 \\
& \beta_{6} \begin{array}{ccc}
\beta_{6}-a & \beta_{7} 4 & \alpha_{5} \\
\alpha_{2} & \alpha_{6} & \alpha_{4}
\end{array} \\
& \beta_{8} 3-a \quad \beta_{9} 3 \\
& \chi^{\alpha_{4}} \quad \alpha_{\alpha_{6}} \quad \chi^{\alpha_{3}} \\
& \beta_{10} 2-a \quad \beta_{11} 2 \\
& \alpha_{\alpha_{5}} \backslash^{\alpha_{3}} / \alpha_{6} \backslash^{\alpha_{1}} \\
& \beta_{12} 1-a \quad \beta_{13} 1-a \quad \beta_{14} 1
\end{aligned}
$$

Let us first consider the regular strata corresponding to $a \geq 5$. Then $\Psi_{\lambda}$ is empty and $\Delta_{\lambda, n}^{+}=\left\{\beta_{0}, \beta_{1}, \beta_{2}, \beta_{3}, \beta_{4}, \beta_{5}, \beta_{7}, \beta_{9}, \beta_{11}, \beta_{14}\right\}$. Taking differences for adjacent $\beta$ we find that the simple roots of $\Delta_{\lambda}^{+}$are $-\beta_{0}, \alpha_{1}, \alpha_{2}, \alpha_{3}, \alpha_{4}, \alpha_{5}$ which give the following Dynkin diagram:

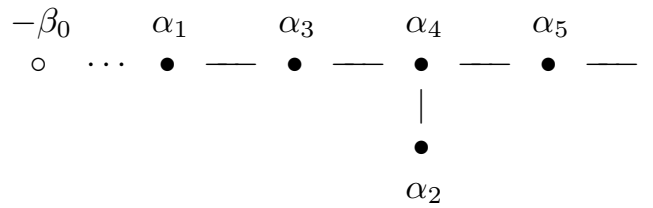


This is the diagram for $S O(2,10)$ and so $\Delta_{\lambda}$ is of type $S O(2,10)$.

Next we consider a different stratum of the same cone, say the stratum corresponding to $a=2$. Then $\Psi_{\lambda}=\left\{ \pm \beta_{10}\right\}$. From the data given in 4.6 we find that the noncompact roots positive at $\lambda+\rho$ and orthogonal to $\beta_{10}$ are:

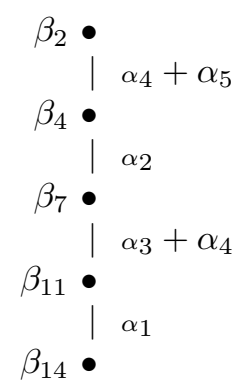

Taking differences for adjacent $\beta$ we obtain the following Dynkin diagram for $\Delta_{\lambda}^{+}$:

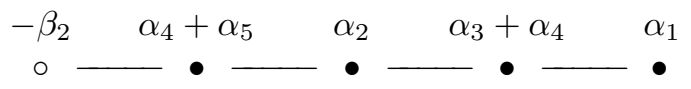

This is the diagram for $S U(1,4)$ and so $\Delta_{\lambda}$ is of type $S U(1,4)$ when $a=2$.

\section{Resolutions And Hilbert series for $E_{6}$}

6.1. In this section we give explicit resolutions and Hilbert series for the unitary highest weight representation of $E_{6}$. We list the representations by cones and their strata as in table 4.9. In each case we first give the general form of a highest weight $\lambda$ in the cone. The nonnegative root numbers, i.e., the values $\left(\lambda+\rho, \beta_{i}^{\vee}\right)$ for $\beta_{i}$ noncompact, are given in diagramatic form as in 4.7. Below this diagram we then list the cases by strata. In each case we first give the singularity type of $\lambda+\rho$. More precisely, we give the parameters $(w, T)$ for the stratum as explained in 4.4. We also list the vertex $v_{w, T}$ as explained in 4.4. Next we give the reduced root system $\Delta_{\lambda}$ which is from the root numbers as in section 5 . We then give the minimal resolution of $L=L_{\lambda}$ by generalized Verma modules as in Corollary 3.8. We write $N_{i}$ for the generalized Verma module of highest weights $\eta_{i}$. All highest weight are explicitly given. Furthermore, we give the degrees of the maps in the resolution. For example,

$$
0 \rightarrow N_{4} \stackrel{+2}{\longrightarrow} N_{3} \stackrel{+1}{\longrightarrow} N_{2} \stackrel{+2}{\longrightarrow} N_{1} \stackrel{+1}{\longrightarrow} N_{0} \rightarrow L \rightarrow 0
$$

would mean that the map from $N_{1}$ to $N_{0}$ is homogenous of degree 1 , the map from $N_{2}$ to $N_{1}$ is homogeneous of degree 2, etc. Here we consider the modules $N_{i}$ and $L$ as graded $S=S\left(\mathfrak{p}^{-}\right)$modules. (For more details see [EH].) We then give the Hilbert series expressed as a rational function $h(t)=P(t) /(1-t)^{d}$ with $P(t) \in \mathbb{Z}[t]$ such that $P(1) \neq 0$. When an explicit expression is too complicated to write down, we express the coefficients simply in terms of the dimensions of the finite dimensional irreducible $\mathfrak{k}$-modules $F_{\eta_{i}}$. We write $d_{i}$ for $\operatorname{dim}\left(F_{\eta_{i}}\right)$. The Gelfand-Kirilov dimension of the representation $L$ is the degree of the denominator of the Hilbert series; the Bernstein degree is the (positive) integer $P(1)$. We give the explicit Hilbert series and Bernstein degree of the module $L=L_{\lambda}$ when $\lambda=\nu_{v, T}$ is the vertex of the stratum. 
6.2. $Q_{\lambda} \simeq S U(1,1), z=1$.

Highest weight: $\lambda=\sum_{i=2}^{6} a_{i} \omega_{i}+\left(-2 a_{2}-2 a_{3}-3 a_{4}-2 a_{5}-a_{6}-10\right) \omega_{1}$ with $a_{2} \geq 1$ and $a_{i} \geq 0$ for $i \neq 2$.

Stratum: $(v, T)=\left(v_{1}, \varnothing\right) \quad(\lambda+\rho$ regular $) \quad$ Vertex: $\omega_{2}-12 \omega_{1}$.

Reduced root system: $\Delta_{\lambda} \simeq S U(1,1), \Delta_{\lambda, n}^{+}=\left\{\beta_{0}\right\}$.

Resolution:

$$
0 \rightarrow N_{1} \stackrel{+1}{\longrightarrow} N_{0} \rightarrow L \rightarrow 0 .
$$

$$
\begin{aligned}
& \eta_{0}=\lambda, \\
& \eta_{1}=\lambda-\beta_{0}=\lambda-\omega_{2} .
\end{aligned}
$$

Hilbert series: $h(t)=\left(d_{0}-d_{1} t\right) /(1-t)^{16}$.

Gelfand-Kirillov dimension: 16 .

Bernstein degree: $d_{0}-d_{1}$.

Hilbert series at vertex: $h(t)=(16-t) /(1-t)^{16}$.

Bernstein degree at vertex: 15 .

6.3. $Q_{\lambda} \simeq S U(1,2), z=2$.

Highest weight: $\lambda=a \omega_{3}+b \omega_{4}+c \omega_{5}+d \omega_{6}+(-2 a-3 b-2 c-d-9) \omega_{1}$ with $b \geq 1$ and $a, c, d \geq 0$.

Stratum: $(v, T)=\left(v_{2}, \varnothing\right) \quad(\lambda+\rho$ regular $) \quad$ Vertex: $\omega_{4}-12 \omega_{1}$.

Reduced root system: $\Delta_{\lambda} \simeq S U(1,2), \Delta_{\lambda, n}^{+}=\left\{\beta_{0}, \beta_{1}\right\}$.

Resolution:

$$
\begin{gathered}
0 \rightarrow N_{2} \stackrel{+1}{\longrightarrow} N_{1} \stackrel{+1}{\longrightarrow} N_{0} \rightarrow L \rightarrow 0 . \\
\eta_{0}=\lambda=a \omega_{3}+b \omega_{4}+c \omega_{5}+d \omega_{6}+(-2 a-3 b-2 c-d-9) \omega_{1}, \\
\eta_{1}=\lambda-\beta_{1}=\omega_{2}+a \omega_{3}+(b-1) \omega_{4}+c \omega_{5}+d \omega_{6}+(-2 a-3 b-2 c-d-9) \omega_{1}, \\
\eta_{2}=\lambda-\beta_{1}-\beta_{0}=a \omega_{3}+(b-1) \omega_{4}+c \omega_{5}+d \omega_{6}+(-2 a-3 b-2 c-d-9) \omega_{1} .
\end{gathered}
$$

Hilbert series: $h(t)=\left(d_{0}-d_{1} t+d_{2} t^{2}\right) /(1-t)^{16}$.

Gelfand-Kirillov dimension: 16 .

Bernstein degree: $d_{0}-d_{1}+d_{2}$.

Hilbert series at vertex: $h(t)=\left(120-16 t+t^{2}\right) /(1-t)^{16}$.

Bernstein degree at vertex: 105.

6.4. $Q_{\lambda} \simeq S U(1,3), z=3$.

Highest weight: $\lambda=a \omega_{3}+b \omega_{5}+c \omega_{6}+(-2 a-2 b-c-8) \omega_{1}$ with $a, b \geq 1$ and $c \geq 0$.

Root numbers:

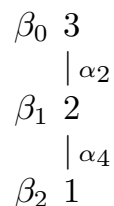

Stratum: $(v, T)=\left(v_{3}, \varnothing\right) \quad(\lambda+\rho$ regular $) \quad$ Vertex: $\omega_{3}+\omega_{5}-12 \omega_{1}$.

Reduced root system: $\Delta_{\lambda} \simeq S U(1,3), \Delta_{\lambda, n}^{+}=\left\{\beta_{0}, \beta_{1}, \beta_{2}\right\}$.

Resolution:

$$
\begin{gathered}
0 \rightarrow N_{3} \stackrel{+1}{\longrightarrow} N_{2} \stackrel{+1}{\longrightarrow} N_{1} \stackrel{+1}{\longrightarrow} N_{0} \rightarrow L \rightarrow 0 \\
\eta_{0}=\lambda=a \omega_{3}+b \omega_{5}+c \omega_{6}+(-2 a-2 b-c-8) \omega_{1} \\
\eta_{1}=\lambda-\beta_{2}=(a-1) \omega_{3}+\omega_{4}+(b-1) \omega_{5}+c \omega_{6}+(-2 a-2 b-c-8) \omega_{1}, \\
\eta_{2}=\lambda-\beta_{2}-\beta_{1}=\omega_{2}+(a-1) \omega_{3}+(b-1) \omega_{5}+c \omega_{6}+(-2 a-2 b-c-8) \omega_{1}, \\
\eta_{3}=\lambda-\beta_{2}-\beta_{1}-\beta_{0}=(a-1) \omega_{3}+(b-1) \omega_{5}+c \omega_{6}+(-2 a-2 b-c-8) \omega_{1} .
\end{gathered}
$$


Hilbert series: $h(t)=\left(d_{0}-d_{1} t+d_{2} t^{2}-d_{3} t^{3}\right) /(1-t)^{16}$.

Gelfand-Kirillov dimension: 16 .

Bernstein degree: $d_{0}-d_{1}+d_{2}-d_{3}$.

Hilbert series at vertex: $h(t)=\left(560-120 t+16 t^{2}-t^{3}\right) /(1-t)^{16}$.

Bernstein degree at vertex: 455 .

6.5. $Q_{\lambda} \simeq S U(1,4)^{(a)}, z=4$.

Highest weight: $\lambda=a \omega_{5}+b \omega_{6}+(-2 a-b-7) \omega_{1}$ with $a \geq 1$ and $b \geq 0$.

Root numbers:

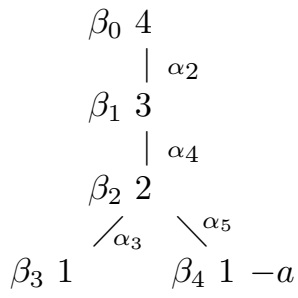

$a>1$ :

Stratum: $(v, T)=\left(v_{4}, \varnothing\right) \quad(\lambda+\rho$ regular $) \quad$ Vertex: $2 \omega_{5}-11 \omega_{1}$.

Reduced root system: $\Delta_{\lambda} \simeq S U(1,4), \Delta_{\lambda, n}^{+}=\left\{\beta_{0}, \beta_{1}, \beta_{2}, \beta_{3}\right\}$.

Resolution:

$$
\begin{gathered}
0 \rightarrow N_{4} \stackrel{+1}{\longrightarrow} N_{3} \stackrel{+1}{\longrightarrow} N_{2} \stackrel{+1}{\longrightarrow} N_{1} \stackrel{+1}{\longrightarrow} N_{0} \rightarrow L \rightarrow 0 . \\
\eta_{0}=\lambda=a \omega_{5}+b \omega_{6}+(-2 a-b-7) \omega_{1}, \\
\eta_{1}=\lambda-\beta_{3}=\omega_{3}+(a-1) \omega_{5}+b \omega_{6}+(-2 a-b-8) \omega_{1}, \\
\eta_{2}=\lambda-\beta_{3}-\beta_{2}=\omega_{4}+(a-2) \omega_{5}+b \omega_{6}+(-2 a-b-8) \omega_{1}, \\
\eta_{3}=\lambda-\beta_{3}-\beta_{2}-\beta_{1}=\omega_{2}+(a-2) \omega_{5}+b \omega_{6}+(-2 a-b-8) \omega_{1}, \\
\eta_{4}=\lambda-\beta_{2}-\beta_{1}-\beta_{1}-\beta_{0}=(a-2) \omega_{5}+b \omega_{6}+(-2 a-b-8) \omega_{1} .
\end{gathered}
$$

Hilbert series: $h(t)=\left(\sum_{i=0}^{4}(-1)^{i} d_{i} t^{i}\right) /(1-t)^{16}$.

Gelfand-Kirillov dimension: 16 .

Bernstein degree: $\sum_{i=0}^{4}(-1)^{i} d_{i}$.

Hilbert series at vertex: $h(t)=\left(770-560+120 t^{2}-16 t^{3}+t^{4}\right) /(1-t)^{16}$.

Bernstein degree at vertex: 315 .

$a=1$ :

Stratum: $(v, T)=\left(v_{6},\left\{\alpha_{3}\right\}\right) \quad(\lambda+\rho$ semi-regular $) \quad$ Vertex: $\omega_{5}-9 \omega_{1}$.

Reduced root system: $\Delta_{\lambda} \simeq S U(1,1), \Delta_{\lambda, n}^{+}=\left\{\beta_{3}\right\}$.

Resolution:

$$
0 \rightarrow N_{1} \stackrel{+1}{\longrightarrow} N_{0} \rightarrow L \rightarrow 0 .
$$

$\eta_{0}=\lambda=\omega_{5}+b \omega_{6}+(-b-9) \omega_{1}$,

$\eta_{1}=\lambda-\beta_{3}=\omega_{3}+b \omega_{6}+(-b-10) \omega_{1}$,

Hilbert series: $h(t)=\left(d_{0}-d_{1} t\right) /(1-t)^{27}$.

Gelfand-Kirillov dimension: 16 .

Bernstein degree: $d_{0}-d_{1}=(4 b+29)(b+7)(b+6)(b+5)(b+4)(b+3)(b+1) / 2520$.

Hilbert series at vertex: $h(t)=(45-16 t) /(1-t)^{16}$.

Bernstein degree at vertex: 29.

6.6. $Q_{\lambda} \simeq S U(1,4)^{(b)}, z=4$.

Highest weight: $\lambda=a \omega_{3}+b \omega_{6}+(-2 a-b-7) \omega_{1}$ with $a, b \geq 1$. 
Root numbers:

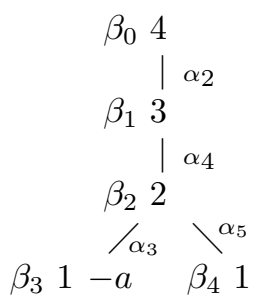

$a>1$ :

Stratum: $(v, T)=\left(v_{5}, \varnothing\right) \quad(\lambda+\rho$ regular $) \quad$ Vertex: $2 \omega_{3}+\omega_{6}-12 \omega_{1}$.

Reduced root system: $\Delta_{\lambda} \simeq S U(1,4), \Delta_{\lambda, n}^{+}=\left\{\beta_{0}, \beta_{1}, \beta_{2}, \beta_{4}\right\}$.

Resolution:

$$
\begin{gathered}
\quad 0 \rightarrow N_{4} \stackrel{+1}{\longrightarrow} N_{3} \stackrel{+1}{\longrightarrow} N_{2} \stackrel{+1}{\longrightarrow} N_{1} \stackrel{+1}{\longrightarrow} N_{0} \rightarrow L \rightarrow 0 . \\
\eta_{0}=\lambda=a \omega_{3}+b \omega_{6}+(-2 a-b-7) \omega_{1}, \\
\eta_{1}=\lambda-\beta_{4}=(a-1) \omega_{3}+\omega_{5}+(b-1) \omega_{6}+(-2 a-b-7) \omega_{1}, \\
\eta_{2}=\lambda-\beta_{4}-\beta_{2}=(a-2) \omega_{3}+\omega_{4}+(b-1) \omega_{6}+(-2 a-b-7) \omega_{1}, \\
\eta_{3}=\lambda-\beta_{4}-\beta_{2}-\beta_{1}=\omega_{2}+(a-2) \omega_{3}+(b-1) \omega_{6}+(-2 a-b-7) \omega_{1}, \\
\eta_{4}=\lambda-\beta_{4}-\beta_{2}-\beta_{1}-\beta_{0}=(a-2) \omega_{3}+(b-1) \omega_{6}+(-2 a-b-7) \omega_{1} .
\end{gathered}
$$

Hilbert series: $h(t)=\left(\sum_{i=0}^{5}(-1)^{i} d_{i} t^{i}\right) /(1-t)^{16}$.

Gelfand-Kirillov dimension: 16 .

Bernstein degree: $\sum_{i=0}^{4}(-1)^{i} d_{i}$.

Hilbert series at vertex: $h(t)=\left(1050-560 t+120 t^{2}-16 t^{3}+t^{4}\right) /(1-t)^{16}$.

Bernstein degree at vertex: 595 .

$a=1$ :

Stratum: $(v, T)=\left(v_{6},\left\{\alpha_{2}\right\}\right) . \quad(\lambda+\rho$ semi-regular $) \quad$ Vertex: $\omega_{3}+\omega_{6}-10 \omega_{1}$.

Reduced root system: $\Delta_{\lambda} \simeq S U(1,1), \Delta_{\lambda, n}^{+}=\left\{\beta_{4}\right\}$.

Resolution:

$$
\begin{aligned}
& 0 \rightarrow N_{1} \stackrel{+1}{\longrightarrow} N_{0} \rightarrow L \rightarrow 0 . \\
& \eta_{0}=\lambda=\omega_{3}+b \omega_{6}+(-b-9) \omega_{1}, \\
& \eta_{1}=\lambda-\beta_{4}=\omega_{5}+(b-1) \omega_{6}+(-b-9) \omega_{1} .
\end{aligned}
$$

Hilbert series: $h(t)=\left(d_{0}-d_{1} t\right) /(1-t)^{16}$.

Gelfand-Kirillov dimension: 16 .

Bernstein degree: $d_{0}-d_{1}=(4 b+7)(b+8)(b+6)(b+5)(b+4)(b+3)(b+2) / 2520$.

Hilbert series at vertex: $h(t)=(144-45 t) /(1-t)^{16}$.

Bernstein degree at vertex: 99.

6.7. $Q_{\lambda} \simeq S U(1,5), z=5$.

Highest weight: $\lambda=a \omega_{3}+(-2 a-6) \omega_{1}$ with $a \geq 1$.

Root numbers:

$$
\begin{aligned}
& \beta_{0} 5 \\
& \mid \alpha_{2} \\
& \beta_{1} 4 \\
& \alpha_{4} \\
& \beta_{2} 3 \\
& / \alpha_{3} \backslash \alpha_{5} \\
& \beta_{3} 2-a \quad \beta_{4} 2 \\
& \searrow^{\alpha_{5}} / \alpha_{3} \quad \chi^{\alpha_{6}} \\
& \beta_{5} 1-a \quad \beta_{6} 1
\end{aligned}
$$


$a>2$ :

Stratum: $(v, T)=\left(v_{5}, \varnothing\right) \quad(\lambda+\rho$ regular $) \quad$ Vertex: $3 \omega_{3}-12 \omega_{1}$.

Reduced root system: $\Delta_{\lambda} \simeq S U(1,5), \Delta_{\lambda, n}^{+}=\left\{\beta_{0}, \beta_{1}, \beta_{2}, \beta_{4}, \beta_{6}\right\}$.

Resolution:

$$
\begin{aligned}
& \quad 0 \rightarrow N_{5} \stackrel{+1}{\longrightarrow} N_{4} \stackrel{+1}{\longrightarrow} N_{3} \stackrel{+1}{\longrightarrow} N_{2} \stackrel{+1}{\longrightarrow} N_{1} \stackrel{+1}{\longrightarrow} N_{0} \rightarrow L \rightarrow 0 . \\
& \eta_{0}=\lambda=a \omega_{3}+(-2 a-6) \omega_{1}, \\
& \eta_{1}=\lambda-\beta_{6}=(a-1) \omega_{3}+\omega_{6}+(-2 a-6) \omega_{1}, \\
& \eta_{2}=\lambda-\beta_{6}-\beta_{4}=(a-2) \omega_{3}+\omega_{5}+(-2 a-6) \omega_{1}, \\
& \eta_{3}=\lambda-\beta_{6}-\beta_{4}-\beta_{2}=(a-3) \omega_{3}+\omega_{4}+(-2 a-6) \omega_{1}, \\
& \eta_{4}=\lambda-\beta_{6}-\beta_{4}-\beta_{2}-\beta_{1}=\omega_{2}+(a-3) \omega_{3}+(-2 a-6) \omega_{1}, \\
& \eta_{5}=\lambda-\beta_{6}-\beta_{4}-\beta_{2}-\beta_{1}-\beta_{0}=(a-3) \omega_{3}+(-2 a-6) \omega_{1} .
\end{aligned}
$$

Hilbert series: $h(t)=\left(\sum_{i=0}^{5}(-1)^{i} d_{i} t^{i}\right) /(1-t)^{16}$.

Gelfand-Kirillov dimension: 16 .

Bernstein degree: $\sum_{i=0}^{6}(-1)^{i} d_{i}=(2 a+5)(a+4)(a+3)(a+2)(a+1) / 120$.

Hilbert series at vertex: $h(t)=\left(672-1050 t+560 t^{2}-120 t^{3}+16 t^{4}-t^{5}\right) /(1-t)^{16}$.

Bernstein degree at vertex: 77 .

$a=2$ :

Stratum: $(v, T)=\left(v_{9},\left\{\alpha_{2}\right\}\right) \quad(\lambda+\rho$ semi-regular $) \quad$ Vertex: $2 \omega_{3}-10 \omega_{1}$.

Reduced root system: $\Delta_{\lambda} \simeq S U(1,2), \Delta_{\lambda, n}^{+}=\left\{\beta_{4}, \beta_{6}\right\}$.

Resolution:

$$
0 \rightarrow N_{2} \stackrel{+1}{\longrightarrow} N_{1} \stackrel{+1}{\longrightarrow} N_{0} \rightarrow L \rightarrow 0 .
$$

$$
\begin{aligned}
& \eta_{0}=\lambda=2 \omega_{3}-10 \omega_{1}, \\
& \eta_{1}=\lambda-\beta_{6}=\omega_{3}+\omega_{6}-10 \omega_{1}, \\
& \eta_{2}=\lambda-\beta_{6}-\beta_{4}=\omega_{5}-10 \omega_{1} .
\end{aligned}
$$

Hilbert series: $h(t)=\left(126-144 t+45 t^{2}\right) /(1-t)^{16}$.

Gelfand-Kirillov dimension: 16.

Bernstein degree: $126-144+45=27$.

$a=1$ :

Stratum: $(v, T)=\left(v_{11},\left\{\alpha_{4}\right\}\right) \quad(\lambda+\rho$ semi-regular $) \quad$ Vertex: $\omega_{3}-8 \omega_{1}$.

Reduced root system: $\Delta_{\lambda} \simeq S U(1,2), \Delta_{\lambda, n}^{+}=\left\{\beta_{2}, \beta_{6}\right\}$.

Resolution:

$$
0 \rightarrow N_{2} \stackrel{+2}{\longrightarrow} N_{1} \stackrel{+1}{\longrightarrow} N_{0} \rightarrow L \rightarrow 0 .
$$

$$
\begin{aligned}
& \eta_{0}=\lambda=\omega_{3}-8 \omega_{1}, \\
& \eta_{1}=\lambda-\beta_{6}=\omega_{6}-8 \omega_{1}, \\
& \eta_{2}=s_{3} s_{5}\left(\lambda+\rho-\beta_{6}-2 \beta_{2}\right)-\rho=-9 \omega_{1} .
\end{aligned}
$$

Hilbert series: $h(t)=\left(16-10 t+t^{3}\right) /(1-t)^{16}$.

Gelfand-Kirillov dimension: 16 .

Bernstein degree: $16-10+1=7$.

6.8. $Q_{\lambda} \simeq S O(2,8), z=4$ (first reduction point).

Highest weight: $\lambda=a \omega_{6}+(-a-7) \omega_{1}$ with $a \geq 1$. 
Root numbers:

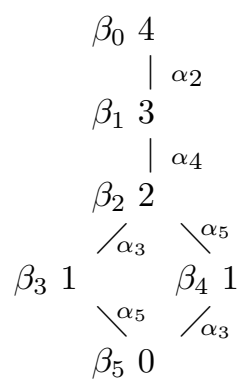

Stratum: $(v, T)=\left(v_{8},\left\{\alpha_{4}\right\}\right) \quad(\lambda+\rho$ semi-regular $) \quad$ Vertex: $\omega_{6}-8 \omega_{1}$.

Reduced root system: $\Delta_{\lambda, n}^{+}=\left\{\beta_{2}\right\}, \Delta_{\lambda} \simeq S U(1,1)$.

Resolution:

$$
0 \rightarrow N_{1} \stackrel{+2}{\longrightarrow} N_{0} \rightarrow L \rightarrow 0 .
$$

$$
\begin{aligned}
& \eta_{0}=\lambda=a \omega_{6}+(-a-7) \omega_{1}, \\
& \eta_{1}=s_{3} s_{5}\left(\lambda+\rho-2 \beta_{2}\right)-\rho=(a-1) \omega_{6}+(-a-8) \omega_{1},
\end{aligned}
$$

Hilbert series: $h(t)=\left(d_{0}-d_{1} t^{2}\right) /(1-t)^{16}$.

Gelfand-Kirillov dimension: 16 .

Bernstein degree: $d_{0}-d_{1}=(2 a+7)(a+6)(a+5)(a+4)(a+3)(a+2)(a+1) / 5040$.

Hilbert series at vertex: $h(t)=\left(10-t^{2}\right) /(1-t)^{16}$.

Bernstein degree at vertex: 9 .

6.9. $Q_{\lambda} \simeq S O(2,8), z=7$ (second reduction point).

Highest weight: $\lambda=a \omega_{6}+(-a-4) \omega_{1}$ with $a \geq 1$.

Root numbers:

$$
\begin{aligned}
& \beta_{0} 7 \\
& \alpha_{2} \\
& \beta_{1} 6 \\
& \text { | } \alpha_{4} \\
& \beta_{2} 5 \\
& / \alpha_{3} \chi^{\alpha_{5}} \\
& \beta_{3} 4 \quad \beta_{4} 4 \\
& \searrow^{\alpha_{5}} / \alpha_{3} \quad \searrow^{\alpha_{6}} \\
& \beta_{5} 3 \quad \beta_{6} 3-a \\
& / \alpha_{4} \backslash \alpha_{6} / \alpha_{3} \\
& \beta_{7} 2 \quad \beta_{8} 2-a \\
& / \alpha_{2} \backslash \alpha_{6} / \alpha_{4} \\
& \beta_{9} 1 \quad \beta_{10} 1-a
\end{aligned}
$$

$a>3$ :

Stratum: $(v, T)=\left(v_{12}, \varnothing\right) \quad(\lambda+\rho$ regular $) \quad$ Vertex: $4 \omega_{6}-8 \omega_{1}$.

Reduced root system: $\Delta_{\lambda} \simeq S U(2,8), \Delta_{\lambda, n}^{+}=\left\{\beta_{0}, \beta_{1}, \beta_{2}, \beta_{3}, \beta_{4}, \beta_{5}, \beta_{7}, \beta_{9}\right\}$.

Resolution:

$$
\begin{aligned}
& 0 \rightarrow N_{8} \stackrel{+1}{\longrightarrow} N_{7} \stackrel{+1}{\longrightarrow} \cdots \stackrel{+1}{\longrightarrow} N_{5} \stackrel{+1}{\longrightarrow} \bigoplus_{N_{4}^{\prime}}^{N_{4}} \stackrel{+1}{\longrightarrow} N_{3} \stackrel{+1}{\longrightarrow} \cdots \stackrel{+1}{\longrightarrow} N_{0} \rightarrow L \rightarrow 0 . \\
& \eta_{0}=\lambda=a \omega_{6}+(-a-4) \omega_{1} \\
& \eta_{1}=\lambda-\beta_{9}=\omega_{2}+(a-1) \omega_{6}+(-a-5) \omega_{1}, \\
& \eta_{2}=\lambda-\beta_{9}-\beta_{7}=\omega_{4}+(a-2) \omega_{6}+(-a-6) \omega_{1},
\end{aligned}
$$




$$
\begin{aligned}
& \eta_{3}=\lambda-\beta_{9}-\beta_{7}-\beta_{5}=\omega_{2}+\omega_{5}+(a-3) \omega_{6}+(-a-7) \omega_{1}, \\
& \eta_{4}=\lambda-\beta_{9}-\beta_{7}-\beta_{5}-\beta_{4}=2 \omega_{5}+(a-4) \omega_{6}+(-a-7) \omega_{1}, \\
& \eta_{4}^{\prime}=\lambda-\beta_{9}-\beta_{7}-\beta_{5}-\beta_{3}=2 \omega_{2}+(a-3) \omega_{6}+(-a-8) \omega_{1}, \\
& \eta_{5}=\lambda-\beta_{9}-\beta_{7}-\beta_{5}-\beta_{4}-\beta_{3}=\omega_{2}+\omega_{5}+(a-4) \omega_{6}+(-a-8) \omega_{1}, \\
& \eta_{6}=\lambda-\beta_{9}-\beta_{7}-\beta_{5}-\beta_{4}-\beta_{3}-\beta_{2}=\omega_{4}+(a-4) \omega_{6}+(-a-8) \omega_{1}, \\
& \eta_{7}=\lambda-\beta_{9}-\beta_{7}-\beta_{5}-\beta_{4}-\beta_{3}-\beta_{2}-\beta_{1}=\omega_{2}+(a-4) \omega_{6}+(-a-8) \omega_{1}, \\
& \eta_{8}=\lambda-\beta_{9}-\beta_{7}-\beta_{5}-\beta_{4}-\beta_{3}-\beta_{2}-\beta_{1}-\beta_{0}=(a-4) \omega_{6}+(-a-8) \omega_{1} .
\end{aligned}
$$

Hilbert series: $h(t)=\left(d_{0}-d_{1} t+\cdots+\left(d_{4}+d_{4}^{\prime}\right) t^{4}-d_{5} t^{5}+\cdots+d_{8} t^{8}\right) /(1-t)^{16}$. Gelfand-Kirillov dimension: 16.

Bernstein degree: 1.

Hilbert series at vertex:

$$
\begin{aligned}
h(t)=( & 600-2640 t+4312 t^{2}-3696 t^{3}+1820 t^{4} \\
& \left.-560 t^{5}+120 t^{6}-16 t^{7}+t^{8}\right) /(1-t)^{16} .
\end{aligned}
$$

$a=3$ :

Stratum: $(v, T)=\left(v_{15},\left\{\alpha_{1}\right\}\right) \quad(\lambda+\rho$ semi-regular $) \quad$ Vertex: $3 \omega_{6}-7 \omega_{1}$.

Reduced root system: $\Delta_{\lambda} \simeq S U(1,4), \Delta_{\lambda, n}^{+}=\left\{\beta_{3}, \beta_{5}, \beta_{7}, \beta_{9}\right\}$.

Resolution:

$$
\begin{aligned}
& \quad 0 \rightarrow N_{4} \stackrel{+1}{\longrightarrow} N_{3} \stackrel{+1}{\longrightarrow} N_{2} \stackrel{+1}{\longrightarrow} N_{1} \stackrel{+1}{\longrightarrow} N_{0} \rightarrow L \rightarrow 0 . \\
& \eta_{0}=\lambda=3 \omega_{6}-7 \omega_{1}, \\
& \eta_{1}=\lambda-\beta_{9}=\omega_{2}+2 \omega_{6}-8 \omega_{1}, \\
& \eta_{2}=\lambda-\beta_{9}-\beta_{7}=\omega_{4}+\omega_{6}-9 \omega_{1}, \\
& \eta_{3}=\lambda-\beta_{9}-\beta_{7}-\beta_{5}=\omega_{2}+\omega_{5}-10 \omega_{1}, \\
& \eta_{4}=\lambda-\beta_{9}-\beta_{7}-\beta_{5}-\beta_{3}=2 \omega_{2}-11 \omega_{1} . \\
& \text { Hilbert series: } h(t)=\left(210-720 t+945 t^{2}-560 t^{3}+126 t^{4}\right) /(1-t)^{16} .
\end{aligned}
$$

Gelfand-Kirillov dimension: 16 .

Bernstein degree: $210-720+945-560+126=1$. $a=2$ :

Stratum: $(v, T)=\left(v_{17},\left\{\alpha_{3}\right\}\right) \quad(\lambda+\rho$ semi-regular $) \quad$ Vertex: $2 \omega_{6}-6 \omega_{1}$.

Reduced root system: $\Delta_{\lambda} \simeq S U(1,4), \Delta_{\lambda, n}^{+}=\left\{\beta_{2}, \beta_{4}, \beta_{7}, \beta_{9}\right\}$.

Resolution:

$$
\begin{aligned}
& \quad 0 \rightarrow N_{4} \stackrel{+1}{\longrightarrow} N_{3} \stackrel{+2}{\longrightarrow} N_{2} \stackrel{+1}{\longrightarrow} N_{1} \stackrel{+1}{\longrightarrow} N_{0} \rightarrow L \rightarrow 0 . \\
& \eta_{0}=\lambda=2 \omega_{6}-6 \omega_{1}, \\
& \eta_{1}=\lambda-\beta_{9}=\omega_{2}+\omega_{6}-7 \omega_{1}, \\
& \eta_{2}=\lambda-\beta_{9}-\beta_{7}=\omega_{4}-8 \omega_{1}, \\
& \eta_{3}=s_{3} s_{6}\left(\lambda+\rho-\beta_{9}-\beta_{7}-2 \beta_{4}\right)-\rho=\omega_{5}-9 \omega_{1}, \\
& \eta_{4}=s_{3} s_{6}\left(\lambda+\rho-\beta_{9}-\beta_{7}-2 \beta_{4}-\beta_{2}\right)-\rho=\omega_{2}-10 \omega_{1} .
\end{aligned}
$$

Hilbert series: $h(t)=\left(54-144 t+120 t^{2}-45 t^{4}+16 t^{5}\right) /(1-t)^{16}$.

Gelfand-Kirillov dimension: 16 .

Bernstein degree: $54-144+120-45+16=1$. $a=1$ :

Stratum: $(v, T)=\left(v_{19},\left\{\alpha_{4}\right\}\right) \quad(\lambda+\rho$ semi-regular $) \quad$ Vertex: $\omega_{6}-5 \omega_{1}$.

Reduced root system: $\Delta_{\lambda} \simeq S U(1,4), \Delta_{\lambda, n}^{+}=\left\{\beta_{1}, \beta_{4}, \beta_{5}, \beta_{9}\right\}$.

Resolution:

$$
\begin{gathered}
0 \stackrel{\rightarrow}{\rightarrow} N_{4} \stackrel{+2}{\longrightarrow} N_{3} \stackrel{+1}{\longrightarrow} N_{2} \stackrel{+2}{\longrightarrow} N_{1} \stackrel{+1}{\longrightarrow} N_{0} \rightarrow L \rightarrow 0 . \\
\eta_{0}=\lambda=\omega_{6}-5 \omega_{1},
\end{gathered}
$$




$$
\begin{aligned}
& \eta_{1}=\lambda-\beta_{9}=\omega_{2}-6 \omega_{1}, \\
& \eta_{2}=s_{4} s_{6}\left(\lambda+\rho-\beta_{9}-2 \beta_{5}\right)-\rho=\omega_{3}-8 \omega_{1}, \\
& \eta_{3}=s_{4} s_{6}\left(\lambda+\rho-\beta_{9}-2 \beta_{5}-\beta_{4}\right)-\rho=\omega_{6}-8 \omega_{1}, \\
& \eta_{4}=s_{3} s_{5} s_{4} s_{6}\left(\lambda+\rho-\beta_{9}-2 \beta_{5}-\beta_{4}-2 \beta_{1}\right)-\rho=-9 \omega_{1} .
\end{aligned}
$$

Hilbert series: $h(t)=\left(10-16 t+16 t^{3}-10 t^{4}+t^{6}\right) /(1-t)^{16}$.

Gelfand-Kirillov dimension: 16.

Bernstein degree: $10-16+16-10+1=1$.

6.10. $Q_{\lambda} \simeq E_{6}, z=8$.

Highest weight: $\lambda=-3 \omega_{1}$.

Root numbers:

$$
\begin{aligned}
& 8 \beta_{0} \\
& \alpha_{2} \\
& 7 \beta_{1} \\
& \alpha_{4} \mid \\
& 6 \beta_{2} \\
& 5 \beta_{3} \alpha_{3} \backslash 5 \beta_{4} \\
& \alpha_{5} \backslash \alpha_{3} / \alpha_{6} \backslash \\
& 4 \beta_{5} \quad 4 \beta_{6} \\
& \alpha_{4} / \alpha_{6} \backslash \alpha_{3} / \\
& 3 \beta_{7} \quad 3 \beta_{8} \\
& \alpha_{2} / \alpha_{6} \backslash \alpha_{4} / \\
& 2 \beta_{9} \quad 2 \beta_{10} \\
& \alpha_{6} \backslash \alpha_{2} / \alpha_{5} \backslash \\
& 1 \beta_{11} \quad 1 \beta_{12} \\
& \alpha_{5} \backslash \alpha_{2} / \\
& 0 \beta_{13}
\end{aligned}
$$

Stratum: $(v, T)=\left(v_{24},\left\{\alpha_{4}\right\}\right) \quad(\lambda+\rho$ semi-regular $) \quad$ Vertex: $-3 \omega_{1}$.

Reduced root system: $\Delta_{\lambda} \simeq S U(1,5), \quad \Delta_{\lambda, n}^{+}=\left\{\beta_{0}, \beta_{2}, \beta_{3}, \beta_{7}, \beta_{10}\right\}$.

Resolution:

$$
\begin{aligned}
& \quad 0 \rightarrow N_{5} \stackrel{+2}{\longrightarrow} N_{4} \stackrel{+1}{\longrightarrow} N_{3} \stackrel{+2}{\longrightarrow} N_{2} \stackrel{+1}{\longrightarrow} N_{1} \stackrel{+2}{\longrightarrow} N_{0} \rightarrow L \rightarrow 0 . \\
& \eta_{0}=\lambda=-3 \omega_{1}, \\
& \eta_{1}=s_{5} s_{2}\left(\lambda+\rho-2 \beta_{10}\right)-\rho=\omega_{6}-5 \omega_{1}, \\
& \eta_{2}=s_{5} s_{2}\left(\lambda+\rho-\beta_{6}-\beta_{4}\right)-\rho=\omega_{2}-6 \omega_{1}, \\
& \eta_{3}=s_{6} s_{4} s_{5} s_{2}\left(\lambda+\rho-\beta_{6}-\beta_{4}-\beta_{2}\right)-\rho=\omega_{3}-8 \omega_{1}, \\
& \eta_{4}=s_{6} s_{4} s_{5} s_{2}\left(\lambda+\rho-\beta_{6}-\beta_{4}-\beta_{2}-\beta_{1}\right)-\rho=\omega_{6}-8 \omega_{1}, \\
& \eta_{5}=s_{5} s_{3} s_{6} s_{4} s_{5} s_{2}\left(\lambda+\rho-\beta_{6}-\beta_{4}-\beta_{2}-\beta_{1}-\beta_{0}\right)-\rho=-9 \omega_{1} . \\
& \text { Hilbert series: } \\
& \qquad h(t)=\left(1-10 t^{2}+16 t^{3}-16 t^{5}+10 t^{6}-t^{8}\right) /(1-t)^{16} \\
& =\left(1+5 t+5 t^{2}+t^{3}\right) /(1-t)^{11} .
\end{aligned}
$$

Gelfand-Kirillov dimension: 11 .

Bernstein degree: 12 .

Special feature: $\lambda+\rho$ is quasi-dominant (Wallach representation).

\section{Resolutions And Hilbert series for $E_{7}$}

7.1. The same remarks apply as in 6.1 . 
7.2. $Q_{\lambda} \simeq S U(1,1), z=1$.

Highest weight $\lambda=\sum_{i=1}^{6} a_{i} \omega_{i}+\left(-2 a_{1}-2 a_{2}-3 a_{3}-4 a_{4}-3 a_{5}-2 a_{6}-16\right) \omega_{7}$ with $a_{1} \geq 1$ and $a_{i} \geq 0$ for $i \neq 1$.

Stratum: $(v, T)=\left(v_{1}, \varnothing\right) \quad(\lambda+\rho$ regular $) \quad$ Vertex: $\omega_{1}-18 \omega_{7}$.

Reduced root system: $\Delta_{\lambda} \simeq S U(1,1), \Delta_{\lambda, n}^{+}=\left\{\beta_{0}\right\}$.

Resolution:

$$
0 \rightarrow N_{1} \stackrel{+1}{\longrightarrow} N_{0} \rightarrow L \rightarrow 0 .
$$

$\eta_{0}=\lambda$,

$\eta_{1}=\lambda-\beta_{0}=\lambda-\omega_{1}$

Hilbert series: $h(t)=\left(d_{0}-d_{1} t\right) /(1-t)^{27}$.

Gelfand-Kirillov dimension: 27.

Bernstein degree: $d_{0}-d_{1}$.

Hilbert series at vertex: $h(t)=(27-t) /(1-t)^{27}$.

Bernstein degree at vertex: 26 .

7.3. $Q_{\lambda} \simeq S U(1,2), z=2$.

Highest weight: $\lambda=\sum_{i=2}^{6} a_{i} \omega_{i}+\left(-2 a_{2}-3 a_{3}-4 a_{4}-3 a_{5}-2 a_{6}-15\right) \omega_{7}$ with $a_{3} \geq 1$ and $a_{i} \geq 0$ for $i \neq 3$.

Stratum: $(v, T)=\left(v_{2}, \varnothing\right) \quad(\lambda+\rho$ regular $) \quad$ Vertex: $\omega_{3}-18 \omega_{7}$.

Reduced root system: $\Delta_{\lambda} \simeq S U(1,2), \Delta_{\lambda, n}^{+}=\left\{\beta_{0}, \beta_{1}\right\}$.

Resolution:

$$
0 \rightarrow N_{2} \stackrel{+1}{\longrightarrow} N_{1} \stackrel{+1}{\longrightarrow} N_{0} \rightarrow L \rightarrow 0 .
$$

$$
\begin{aligned}
& \eta_{0}=\lambda, \\
& \eta_{1}=\lambda-\beta_{1}=\lambda+\omega_{1}-\omega_{3}, \\
& \eta_{2}=\lambda-\beta_{1}-\beta_{0}=\lambda-\omega_{3} .
\end{aligned}
$$

Hilbert series: $h(t)=\left(d_{0}-d_{1} t+d_{2} t^{2}\right) /(1-t)^{27}$.

Gelfand-Kirillov dimension: 27.

Bernstein degree: $d_{0}-d_{1}+d_{2}$.

Hilbert series at vertex: $h(t)=\left(351-27 t+t^{2}\right) /(1-t)^{27}$.

Bernstein degree at vertex: 325 .

7.4. $Q_{\lambda} \simeq S U(1,3), z=3$.

Highest weight: $\lambda=a \omega_{2}+b \omega_{4}+c \omega_{5}+d \omega_{6}+(-2 a-4 b-3 c-2 d-14) \omega_{7}$ with $b \geq 1$ and $a, c, d \geq 0$.

Stratum: $(v, T)=\left(v_{3}, \varnothing\right) \quad(\lambda+\rho$ regular $) \quad$ Vertex: $\omega_{4}-18 \omega_{7}$.

Reduced root system: $\Delta_{\lambda} \simeq S U(1,3), \Delta_{\lambda, n}^{+}=\left\{\beta_{0}, \beta_{1}, \beta_{2}\right\}$.

Resolution :

$$
0 \rightarrow N_{3} \stackrel{+1}{\longrightarrow} N_{2} \stackrel{+1}{\longrightarrow} N_{1} \stackrel{+1}{\longrightarrow} N_{0} \rightarrow L \rightarrow 0 .
$$

$$
\begin{aligned}
& \eta_{0}=\lambda, \\
& \eta_{1}=\lambda-\beta_{2}=\lambda+\omega_{3}-\omega_{4}, \\
& \eta_{2}=\lambda-\beta_{2}-\beta_{1}=\lambda+\omega_{1}-\omega_{4}, \\
& \eta_{3}=\lambda-\beta_{2}-\beta_{1}-\beta_{0}=\lambda-\omega_{4} .
\end{aligned}
$$

Hilbert series: $h(t)=\left(d_{0}-d_{1} t+d_{2} t^{2}+d_{3} t^{3}\right) /(1-t)^{27}$.

Gelfand-Kirillov dimension: 27.

Bernstein degree: $d_{0}-d_{1}+d_{2}-d_{3}$.

Hilbert series at vertex: $h(t)=\left(2925-351 t+27 t^{2}+t^{3}\right) /(1-t)^{27}$.

Bernstein degree at vertex: 2600 . 
7.5. $Q_{\lambda} \simeq S U(1,4), z=4$.

Highest weight: $\lambda=a \omega_{2}+b \omega_{5}+c \omega_{6}+(-2 a-3 b-2 c-13) \omega_{7}$ with $a, b \geq 1$ and $c \geq 0$.

Stratum: $(v, T)=\left(v_{4}, \varnothing\right) \quad(\lambda+\rho$ regular $) \quad$ Vertex: $\omega_{2}+\omega_{5}-18 \omega_{7}$.

Reduced root system: $\Delta_{\lambda} \simeq S U(1,4), \Delta_{\lambda, n}^{+}=\left\{\beta_{0}, \beta_{1}, \beta_{2}, \beta_{3}\right\}$.

Resolution:

$$
\begin{aligned}
& \quad 0 \rightarrow N_{4} \stackrel{+1}{\longrightarrow} N_{3} \stackrel{+1}{\longrightarrow} N_{2} \stackrel{+1}{\longrightarrow} N_{1} \stackrel{+1}{\longrightarrow} N_{0} \rightarrow L \rightarrow 0 \\
& \eta_{0}=\lambda, \\
& \eta_{1}=\lambda-\beta_{3}=\lambda-\omega_{2}+\omega_{4}-\omega_{5} \\
& \eta_{2}=\lambda-\beta_{3}-\beta_{2}=\lambda-\omega_{2}+\omega_{3}-\omega_{5}, \\
& \eta_{3}=\lambda-\beta_{3}-\beta_{2}-\beta_{1}=\lambda+\omega_{1}-\omega_{2}-\omega_{5} \\
& \eta_{4}=\lambda-\beta_{3}-\beta_{2}-\beta_{1}-\beta_{0}=\lambda-\omega_{2}-\omega_{5} . \\
& \text { Hilbert series: } h(t)=\left(d_{0}-d_{1} t+d_{2} t^{2}-d_{3} t^{3}+d_{4} t^{4}\right) /(1-t)^{27} .
\end{aligned}
$$

Gelfand-Kirillov dimension: 27.

Bernstein degree: $d_{0}-d_{1}+d_{2}-d_{3}+d_{4}$.

Hilbert series at vertex: $h(t)=\left(17550-2925 t+351 t^{2}-27 t^{3}+t^{4}\right) /(1-t)^{27}$.

Bernstein degree at vertex: 14950.

7.6. $Q_{\lambda} \simeq S U(1,5)^{(b)}, z=5$.

Highest weight: $\lambda=a \omega_{2}+b \omega_{6}+(-2 a-2 b-12) \omega_{7}$ with $a, b \geq 1$.

Root numbers:

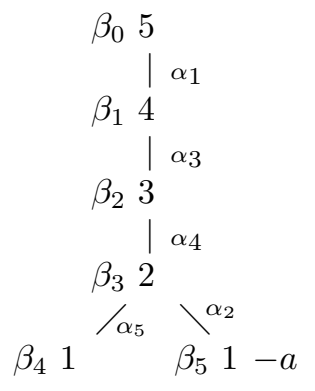

$a>1$ :

Stratum: $(v, T)=\left(v_{6}, \varnothing\right) \quad(\lambda+\rho$ regular $) \quad$ Vertex: $2 \omega_{2}+\omega_{6}-18 \omega_{7}$.

Reduced root system: $\Delta_{\lambda} \simeq S U(1,5), \Delta_{\lambda, n}^{+}=\left\{\beta_{0}, \beta_{1}, \beta_{2}, \beta_{3}, \beta_{4}\right\}$.

Resolution:

$$
\begin{aligned}
& \quad 0 \rightarrow N_{5} \stackrel{+1}{\longrightarrow} N_{4} \stackrel{+1}{\longrightarrow} N_{3} \stackrel{+1}{\longrightarrow} N_{2} \stackrel{+1}{\longrightarrow} N_{1} \stackrel{+1}{\longrightarrow} N_{0} \rightarrow L \rightarrow 0 . \\
& \eta_{0}=\lambda=a \omega_{2}+b \omega_{6}+(-2 a-2 b-12) \omega_{7}, \\
& \eta_{1}=\lambda-\beta_{4}=(a-1) \omega_{2}+\omega_{5}+(b-1) \omega_{6}+(-2 a-2 b-12) \omega_{7}, \\
& \eta_{2}=\lambda-\beta_{4}-\beta_{3}=(a-2) \omega_{2}++\omega_{4}+(b-1) \omega_{6}+(-2 a-2 b-12) \omega_{7}, \\
& \eta_{3}=\lambda-\beta_{4}-\beta_{3}-\beta_{2}=(a-2) \omega_{2}+\omega_{3}+(b-1) \omega_{6}+(-2 a-2 b-12) \omega_{7}, \\
& \eta_{4}=\lambda-\beta_{4}-\beta_{3}-\beta_{2}-\beta_{1}=\omega_{1}+(a-2) \omega_{2}+(b-1) \omega_{6}+(-2 a-2 b-12) \omega_{7}, \\
& \eta_{5}=\lambda-\beta_{4}-\beta_{3}-\beta_{2}-\beta_{1}-\beta_{0}=(a-2) \omega_{2}+(b-1) \omega_{6}+(-2 a-2 b-12) \omega_{7} .
\end{aligned}
$$

Hilbert series: $h(t)=\left(\sum_{i=0}^{5}(-1)^{i} d_{i} t^{i}\right) /(1-t)^{27}$.

Gelfand-Kirillov dimension: 27.

Bernstein degree: $\sum_{i=0}^{5}(-1)^{i} d_{i}$.

Hilbert series at vertex:

$$
h(t)=\left(46332-17550 t+2925 t^{3}-351 t^{3}+27 t^{4}-t^{5}\right) /(1-t)^{27} .
$$


Bernstein degree at vertex: 31382 .

$a=1$ :

Stratum: $(v, T)=\left(v_{7},\left\{\alpha_{2}\right\}\right) \quad(\lambda+\rho$ semi-regular $) \quad$ Vertex: $\omega_{2}+\omega_{6}-16 \omega_{7}$.

Reduced root system: $\Delta_{\lambda} \simeq S U(1,1), \Delta_{\lambda, n}^{+}=\left\{\beta_{4}\right\}$.

Resolution:

$$
\begin{gathered}
0 \rightarrow N_{1} \stackrel{+1}{\longrightarrow} N_{0} \rightarrow L \rightarrow 0 . \\
\eta_{0}=\lambda=\omega_{2}+b \omega_{6}+(-2 b-14) \omega_{7}, \\
\eta_{1}=\lambda-\beta_{4}=\omega_{5}+(b-1) \omega_{6}+(-2 b-14) \omega_{7} .
\end{gathered}
$$

Hilbert series: $h(t)=\left(d_{0}-d_{1} t\right) /(1-t)^{27}$.

Gelfand-Kirillov dimension: 27.

Bernstein degree: $d_{0}-d_{1}$.

Hilbert series at vertex: $h(t)=(1728-351 t) /(1-t)^{27}$.

Bernstein degree at vertex: 1377.

7.7. $Q_{\lambda} \simeq S U(1,5)^{(a)}, z=5$.

Highest weight: $\lambda=a \omega_{5}+b \omega_{6}+(-3 a-2 b-12) \omega_{7}$ with $a \geq 1$ and $b \geq 0$.

Root numbers:

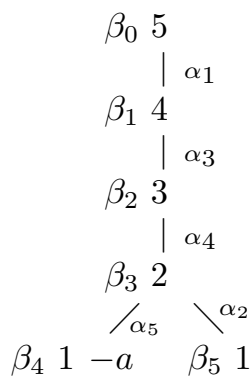

$a>1$ :

Stratum: $(v, T)=\left(v_{5}, \varnothing\right) \quad(\lambda+\rho$ regular $) \quad$ Vertex: $2 \omega_{5}-18 \omega_{7}$.

Reduced root system: $\Delta_{\lambda} \simeq S U(1,5), \Delta_{\lambda, n}^{+}=\left\{\beta_{0}, \beta_{1}, \beta_{2}, \beta_{3}, \beta_{5}\right\}$.

Resolution:

$$
\begin{gathered}
\quad 0 \rightarrow N_{5} \stackrel{+1}{\longrightarrow} N_{4} \stackrel{+1}{\longrightarrow} N_{3} \stackrel{+1}{\longrightarrow} N_{2} \stackrel{+1}{\longrightarrow} N_{1} \stackrel{+1}{\longrightarrow} N_{0} \rightarrow L \rightarrow 0 . \\
\eta_{0}=\lambda=a \omega_{5}+b \omega_{6}+(-3 a-2 b-12) \omega_{7}, \\
\eta_{1}=\lambda-\beta_{5}=\omega_{2}+(a-1) \omega_{5}+b \omega_{6}+(-3 a-2 b-12) \omega_{7}, \\
\eta_{2}=\lambda-\beta_{5}-\beta_{3}=\omega_{4}+(a-2) \omega_{5}+b \omega_{6}+(-3 a-2 b-12) \omega_{7}, \\
\eta_{3}=\lambda-\beta_{5}-\beta_{3}-\beta_{2}=\omega_{3}+(a-2) \omega_{5}+b \omega_{6}+(-3 a-2 b-12) \omega_{7}, \\
\eta_{4}=\lambda-\beta_{5}-\beta_{3}-\beta_{2}-\beta_{1}=\omega_{1}+(a-2) \omega_{5}+b \omega_{6}+(-3 a-2 b-12) \omega_{7}, \\
\eta_{5}=\lambda-\beta_{5}-\beta_{3}-\beta_{2}-\beta_{1}-\beta_{0}=(a-2) \omega_{5}+b \omega_{6}+(-3 a-2 b-12) \omega_{7} .
\end{gathered}
$$

Hilbert series: $h(t)=\left(\sum_{i=0}^{5}(-1)^{i} d_{i} t^{i}\right) /(1-t)^{27}$.

Gelfand-Kirillov dimension: 27.

Bernstein degree: $\sum_{i=0}^{5}(-1)^{i} d_{i}$.

Hilbert series at vertex:

$$
h(t)=\left(34398-17550 t+2925 t^{3}-351 t^{3}+27 t^{4}-t^{5}\right) /(1-t)^{27} .
$$

Bernstein degree at vertex: 19448.

$a=1$ :

Stratum: $(v, T)=\left(v_{7},\left\{\alpha_{3}\right\}\right) \quad(\lambda+\rho$ semi-regular $) \quad$ Vertex: $\omega_{5}-15 \omega_{7}$.

Reduced root system: $\Delta_{\lambda} \simeq S U(1,1), \Delta_{\lambda, n}^{+}=\left\{\beta_{5}\right\}$. 
Resolution:

$$
\begin{aligned}
& 0 \rightarrow N_{1} \stackrel{+1}{\longrightarrow} N_{0} \rightarrow L \rightarrow 0 . \\
& \eta_{0}=\lambda=\omega_{5}+b \omega_{6}+(-2 b-15) \omega_{7}, \\
& \eta_{1}=\lambda-\beta_{5}=\omega_{2}+b \omega_{6}+(-2 b-15) \omega_{7} .
\end{aligned}
$$

Hilbert series: $h(t)=\left(d_{0}-d_{1} t\right) /(1-t)^{27}$.

Gelfand-Kirillov dimension: 27 .

Bernstein degree:

$$
d_{0}-d_{1}=(b+13)(b+11)_{9}(b+9)(b+7)^{2}(b+5)(b+1) /(11)_{9}(7)_{3} .
$$

Hilbert series at vertex: $h(t)=(351-78 t) /(1-t)^{27}$.

Bernstein degree at vertex: 273.

7.8. $Q_{\lambda} \simeq S U(1,6), z=6$.

Highest weight: $\lambda=a \omega_{2}+(-2 a-11) \omega_{7}$ with $a \geq 1$.

Root numbers:

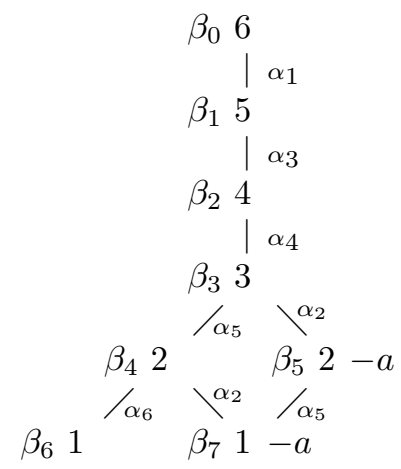

$a>2$ :

Stratum: $(v, T)=\left(v_{8}, \varnothing\right) \quad(\lambda+\rho$ regular $) \quad$ Vertex: $3 \omega_{2}-17 \omega_{7}$. Reduced root system: $\Delta_{\lambda} \simeq S U(1,6), \Delta_{\lambda, n}^{+}=\left\{\beta_{0}, \beta_{1}, \beta_{2}, \beta_{3}, \beta_{4}, \beta_{6}\right\}$.

Resolution:

$$
\begin{aligned}
& \quad 0 \rightarrow N_{6} \stackrel{+1}{\longrightarrow} N_{5} \stackrel{+1}{\longrightarrow} \cdots \stackrel{+1}{\longrightarrow} N_{1} \stackrel{+1}{\longrightarrow} N_{0} \rightarrow L \rightarrow 0 . \\
& \eta_{0}=\lambda=a \omega_{2}+(-2 a-11) \omega_{7}, \\
& \eta_{1}=\lambda-\beta_{6}=(a-1) \omega_{2}+\omega_{6}+(-2 a-12) \omega_{7}, \\
& \eta_{2}=\lambda-\beta_{6}-\beta_{4}=(a-2) \omega_{2}+\omega_{5}+(-2 a-12) \omega_{7}, \\
& \eta_{3}=\lambda-\beta_{6}-\beta_{4}-\beta_{3}=(a-3) \omega_{2}+\omega_{4}+(-2 a-12) \omega_{7}, \\
& \eta_{4}=\lambda-\beta_{6}-\beta_{4}-\beta_{3}-\beta_{2}=(a-3) \omega_{2}+\omega_{3}+(-2 a-12) \omega_{7}, \\
& \eta_{5}=\lambda-\beta_{6}-\beta_{4}-\beta_{3}-\beta_{2}-\beta_{1}=\omega_{1}+(a-3) \omega_{2}+(-2 a-12) \omega_{7}, \\
& \eta_{6}=\lambda-\beta_{6}-\beta_{4}-\beta_{3}-\beta_{2}-\beta_{1}-\beta_{0}=(a-3) \omega_{2}+(-2 a-12) \omega_{7} .
\end{aligned}
$$

Hilbert series: $h(t)=\left(\sum_{i=0}^{6}(-1)^{i} d_{i} t^{i}\right) /(1-t)^{27}$.

Gelfand-Kirillov dimension: 27

Bernstein degree:

$$
\begin{aligned}
\sum_{i=0}^{6}(-1)^{i} d_{i}= & (2 a+11)(2 a+9)(2 a+7)(2 a+5)(a+7)(a+6) \\
& \cdot(a+5)^{2}(a+4)^{3}(a+3)^{2}(a+2)(a+1) /(11 !)(7)(5)(3) .
\end{aligned}
$$

Hilbert series at vertex:

$$
h(t)=\left(43758-46332 t+17550 t^{2}-2925 t^{3}+351 t^{4}-27 t^{5}+t^{6}\right) /(1-t)^{27} .
$$

Bernstein degree at vertex: 12376 . 
$a=2$ :

Stratum: $(v, T)=\left(v_{10},\left\{\alpha_{2}\right\}\right) \quad(\lambda+\rho$ semi-regular $) \quad$ Vertex: $2 \omega_{2}-15 \omega_{7}$.

Reduced root system: $\Delta_{\lambda} \simeq S U(1,2), \Delta_{\lambda, n}^{+}=\left\{\beta_{4}, \beta_{6}\right\}$.

Resolution:

$$
0 \rightarrow N_{2} \stackrel{+1}{\longrightarrow} N_{1} \stackrel{+1}{\longrightarrow} N_{0} \rightarrow L \rightarrow 0 .
$$

$\eta_{0}=\lambda=2 \omega_{2}-15 \omega_{7}$,

$\eta_{1}=\lambda-\beta_{6}=\omega_{2}+\omega_{6}-16 \omega_{7}$,

$\eta_{2}=\lambda-\beta_{6}-\beta_{4}=\omega_{5}-16 \omega_{7}$.

Hilbert series: $h(t)=\left(2430-1728 t+351 t^{2}\right) /(1-t)^{27}$.

Gelfand-Kirillov dimension: 27.

Bernstein degree: $2430-1728+351=1053$.

$a=1$ :

Stratum: $(v, T)=\left(v_{12},\left\{\alpha_{4}\right\}\right) \quad(\lambda+\rho$ semi-regular $) \quad$ Vertex: $\omega_{2}-13 \omega_{7}$.

Reduced root system: $\Delta_{\lambda} \simeq S U(1,2), \Delta_{\lambda, n}^{+}=\left\{\beta_{3}, \beta_{6}\right\}$.

Resolution:

$$
\begin{aligned}
& \quad 0 \rightarrow N_{2} \stackrel{+2}{\longrightarrow} N_{1} \stackrel{+1}{\longrightarrow} N_{0} \rightarrow L \rightarrow 0 . \\
& \eta_{0}=\lambda=\omega_{2}-13 \omega_{7}, \\
& \eta_{1}=\lambda-\beta_{6}=\omega_{6}-14 \omega_{7}, \\
& \eta_{2}=s_{2} s_{5}\left(\lambda+\rho-\beta_{6}-2 \beta_{3}\right)-\rho=-14 \omega_{7} .
\end{aligned}
$$

Hilbert series: $h(t)=\left(78-27 t+t^{3}\right) /(1-t)^{27}$.

Gelfand-Kirillov dimension: 27.

Bernstein degree: $78-27+1=52$.

7.9. $Q_{\lambda} \simeq S O(2,10), z=5$ (first reduction point).

Highest weight: $\lambda=a \omega_{6}+(-2 a-12) \omega_{7}$ with $a \geq 1$.

Root numbers:

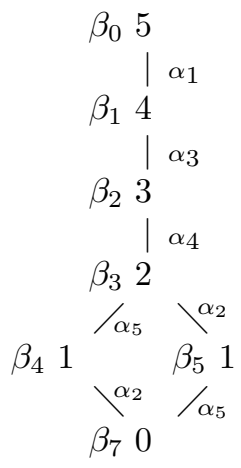

Stratum: $(v, T)=\left(v_{9},\left\{\alpha_{4}\right\}\right) \quad(\lambda+\rho$ semi-regular $) \quad$ Vertex: $\omega_{6}-14 \omega_{7}$.

Reduced root system: $\Delta_{\lambda} \simeq S U(1,1), \Delta_{\lambda, n}^{+}=\left\{\beta_{3}\right\}$.

Resolution:

$$
\begin{gathered}
0 \rightarrow N_{1} \stackrel{+2}{\longrightarrow} N_{0} \rightarrow L \rightarrow 0 . \\
\eta_{0}=\lambda=a \omega_{6}+(-2 a-12) \omega_{7}, \\
\eta_{1}=s_{2} s_{5}\left(\lambda+\rho-2 \beta_{3}\right)-\rho=(a-1) \omega_{6}+(-2 a-12) \omega_{7} .
\end{gathered}
$$

Hilbert series: $h(t)=\left(d_{0}-d_{1} t^{2}\right) /(1-t)^{27}$.

Gelfand-Kirillov dimension: 27.

Bernstein degree: $d_{0}-d_{1}=(2 a+11)(a+10)_{10}(a+7)_{4} /(11)_{10}(7)_{4}$.

Hilbert series at vertex: $h(t)=\left(27-t^{2}\right) /(1-t)^{27}$.

Bernstein degree at vertex: 26. 
7.10. $Q_{\lambda} \simeq S O(2,10), z=9$ (second reduction point).

Highest weight: $\lambda=a \omega_{6}+(-2 a-8) \omega_{7}$ with $a \geq 1$.

Root numbers:

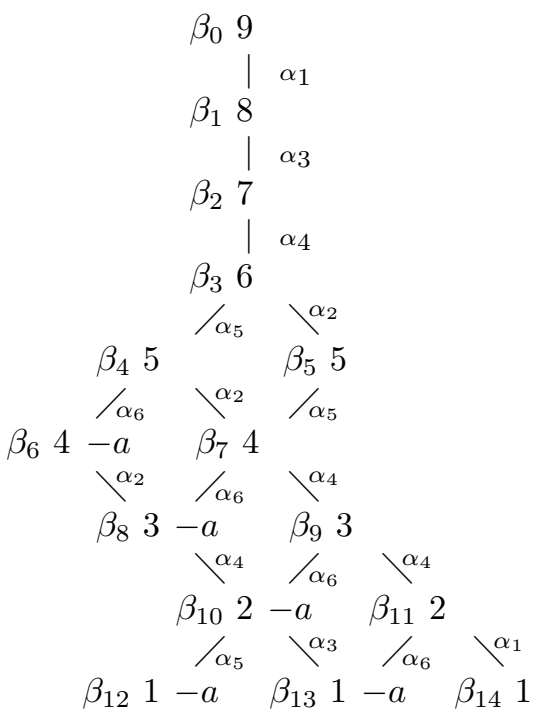

$a>4$ :

Stratum: $(v, T)=\left(v_{16}, \varnothing\right) \quad(\lambda+\rho$ regular $) \quad$ Vertex: $5 \omega_{6}-18 \omega_{7}$.

Reduced root system: $\Delta_{\lambda} \simeq S O(2,10)$,

$\Delta_{\lambda, n}^{+}=\left\{\beta_{0}, \beta_{1}, \beta_{2}, \beta_{3}, \beta_{4}, \beta_{5}, \beta_{7}, \beta_{9}, \beta_{11}, \beta_{14}\right\}$.

Resolution:

$$
\begin{aligned}
& 0 \rightarrow N_{10} \stackrel{+1}{\longrightarrow} N_{9} \stackrel{+1}{\longrightarrow} N_{8} \stackrel{+1}{\longrightarrow} N_{7} \stackrel{+1}{\longrightarrow} N_{6} \stackrel{(+1,+1)}{\longrightarrow} N_{5}^{\prime} \oplus N_{5}^{\prime \prime} \stackrel{(+1,+1)}{\longrightarrow} \\
& \quad N_{4} \stackrel{+1}{\longrightarrow} N_{3} \stackrel{+1}{\longrightarrow} N_{2} \stackrel{+1}{\longrightarrow} N_{1} \stackrel{+1}{\longrightarrow} N_{0} \rightarrow L \rightarrow 0 . \\
& \eta_{0}=\lambda=a \omega_{6}+(-a-8) \omega_{7}, \\
& \eta_{1}=\lambda-\beta_{14}=\omega_{1}+(a-1) \omega_{6}+(-a-8) \omega_{7}, \\
& \eta_{2}=\lambda-\beta_{14}-\beta_{11}=\omega_{3}+(a-2) \omega_{6}+(-a-8) \omega_{7}, \\
& \eta_{3}=\lambda-\beta_{14}-\beta_{11}-\beta_{9}=\omega_{4}+(a-3) \omega_{6}+(-a-8) \omega_{7}, \\
& \eta_{4}=\lambda-\beta_{14}-\beta_{11}-\beta_{9}-\beta_{7}=\omega_{2}+\omega_{5}+(a-4) \omega_{6}+(-a-8) \omega_{7}, \\
& \eta_{5}^{\prime}=\lambda-\beta_{14}-\beta_{11}-\beta_{9}-\beta_{7}-\beta_{5}=2 \omega_{2}+(a-4) \omega_{6}+(-a-8) \omega_{7}, \\
& \eta_{5}^{\prime \prime}=\lambda-\beta_{14}-\beta_{11}-\beta_{9}-\beta_{7}-\beta_{4}=2 \omega_{5}+(a-5) \omega_{6}+(-a-8) \omega_{7}, \\
& \eta_{6}=\lambda-\beta_{14}-\beta_{11}-\beta_{9}-\beta_{7}-\beta_{5}-\beta_{4}=\omega_{2}+\omega_{5}+(a-5) \omega_{6}+(-a-8) \omega_{7}, \\
& \eta_{7}=\lambda-\beta_{14}-\beta_{11}-\cdots-\beta_{7}-\beta_{5}-\beta_{4}-\beta_{3}=\omega_{4}+(a-5) \omega_{6}+(-a-8) \omega_{7}, \\
& \eta_{8}=\lambda-\beta_{14}-\beta_{11}-\cdots-\beta_{5}-\beta_{4}-\beta_{3}-\beta_{2}=\omega_{3}+(a-5) \omega_{6}+(-a-8) \omega_{7}, \\
& \eta_{9}=\lambda-\beta_{14}-\beta_{11}-\cdots-\beta_{4}-\beta_{3}-\beta_{2}-\beta_{1}=\omega_{1}+(a-5) \omega_{6}+(-a-8) \omega_{7}, \\
& \eta_{10}=\lambda-\beta_{14}-\beta_{11}-\cdots-\beta_{3}-\beta_{2}-\beta_{1}-\beta_{0}=(a-5) \omega_{6}+(-a-8) \omega_{7} .
\end{aligned}
$$

Hilbert series:

$$
h(t)=\left(\sum_{i=0}^{10}(-1)^{i} d_{i} t^{i}\right) /(1-t)^{27}=\left(\sum_{i=0}^{9} e_{i} t^{i}\right) /(1-t)^{26},
$$

where $d_{i}=\operatorname{dim}\left(F_{\eta_{i}}\right)$ for $i \neq 5$ and $d_{5}=\operatorname{dim}\left(F_{\eta_{5}^{\prime}}\right)+\operatorname{dim}\left(F_{\eta_{5}^{\prime \prime}}\right)$.

Gelfand-Kirillov dimension: 26.

Bernstein degree:

$$
\sum_{i=0}^{9} e_{i}=\left(2 a^{2}+9 a+7\right)(a+6)(a+5)(a+4)(a+3)(a+2) / 1680 .
$$


Hilbert series at vertex:

$$
\begin{aligned}
h(t)= & \left(100386-371800 t+579150 t^{2}-494208 t^{3}+252252 t^{4}-80730 t^{5}\right. \\
& \left.+17550 t^{6}-2925 t^{7}+351 t^{8}-27 t^{9}+t^{10}\right) /(1-t)^{27} \\
= & \left(100386-271414 t+307736 t^{2}-186472 t^{3}+65780 t^{4}-14950 t^{5}\right. \\
& \left.+2600 t^{6}-325 t^{7}+26 t^{8}-t^{9}\right) /(1-t)^{26} .
\end{aligned}
$$

Bernstein degree at vertex: 3366 .

$a=4$ :

Stratum: $(v, T)=\left(v_{19},\left\{\alpha_{1}\right\}\right) \quad(\lambda+\rho$ semi-regular $) \quad$ Vertex: $4 \omega_{6}-16 \omega_{7}$.

Reduced root system: $\Delta_{\lambda} \simeq S U(1,5), \Delta_{\lambda, n}^{+}=\left\{\beta_{5}, \beta_{7}, \beta_{9}, \beta_{11}, \beta_{14}\right\}$.

Resolution:

$$
\begin{aligned}
& \quad 0 \rightarrow N_{5} \stackrel{+1}{\longrightarrow} N_{4} \stackrel{+1}{\longrightarrow} N_{3} \stackrel{+1}{\longrightarrow} N_{2} \stackrel{+1}{\longrightarrow} N_{1} \stackrel{+1}{\longrightarrow} N_{0} \rightarrow L \rightarrow 0 . \\
& \eta_{0}=\lambda=4 \omega_{6}-16 \omega_{7}, \\
& \eta_{1}=\lambda-\beta_{14}=\omega_{1}+3 \omega_{6}-16 \omega_{7}, \\
& \eta_{2}=\lambda-\beta_{14}-\beta_{11}=\omega_{3}+2 \omega_{6}-16 \omega_{7}, \\
& \eta_{3}=\lambda-\beta_{14}-\beta_{11}-\beta_{9}=\omega_{4}+\omega_{6}-16 \omega_{7}, \\
& \eta_{4}=\lambda-\beta_{14}-\beta_{11}-\beta_{9}-\beta_{7}=\omega_{2}+\omega_{5}-16 \omega_{7}, \\
& \eta_{5}=\lambda-\beta_{14}-\beta_{11}-2 \beta_{9}-\beta_{7}-\beta_{5}=2 \omega_{2}-16 \omega_{7} .
\end{aligned}
$$

Hilbert series:

$$
\begin{aligned}
h(t) & =\left(19305-61425 t+78975 t^{2}-51975 t^{3}+17550 t^{4}-2430 t^{5}\right) /(1-t)^{27} \\
& =\left(19305-42120 t+36855 t^{2}-15120 t^{3}+2430 t^{4}\right) /(1-t)^{26} .
\end{aligned}
$$

Gelfand-Kirillov dimension: 26.

Bernstein degree: $19305-42120+36855-15120+2430=1350$. $a=3$ :

Stratum: $(v, T)=\left(v_{22},\left\{\alpha_{3}\right\}\right) \quad(\lambda+\rho$ semi-regular $) \quad$ Vertex: $3 \omega_{6}-14 \omega_{7}$.

Reduced root system: $\Delta_{\lambda} \simeq S U(1,5), \Delta_{\lambda, n}^{+}=\left\{\beta_{3}, \beta_{4}, \beta_{9}, \beta_{11}, \beta_{14}\right\}$.

Resolution:

$$
\begin{aligned}
& \quad \quad \quad \stackrel{\rightarrow}{\rightarrow} N_{5} \stackrel{+1}{\longrightarrow} N_{4} \stackrel{+2}{\longrightarrow} N_{3} \stackrel{+1}{\longrightarrow} N_{2} \stackrel{+1}{\longrightarrow} N_{1} \stackrel{+1}{\longrightarrow} N_{0} \rightarrow L \rightarrow 0 \\
& \eta_{0}=\lambda=3 \omega_{6}-14 \omega_{7}, \\
& \eta_{1}=\lambda-\beta_{14}=\omega_{1}+2 \omega_{6}-14 \omega_{7}, \\
& \eta_{2}=\lambda-\beta_{14}-\beta_{11}=\omega_{3}+\omega_{6}-14 \omega_{7}, \\
& \eta_{3}=\lambda-\beta_{14}-\beta_{11}-\beta_{9}=\omega_{4}-14 \omega_{7}, \\
& \eta_{4}=s_{2} s_{6}\left(\lambda+\rho-\beta_{14}-\cdots-2 \beta_{4}\right)-\rho=\omega_{5}-15 \omega_{7}, \\
& \eta_{5}=s_{2} s_{6}\left(\lambda+\rho-\beta_{14}-\cdots-\beta_{3}\right)-\rho=\omega_{2}-15 \omega_{7} .
\end{aligned}
$$

Hilbert series:

$$
\begin{aligned}
h(t) & =\left(3003-7722 t+7371 t^{2}-2925 t^{3}+351 t^{5}-78 t^{6}\right) /(1-t)^{27} \\
& =\left(3003-4719 t+2652 t^{2}-273 t^{3}-273 t^{4}+78 t^{5}\right) /(1-t)^{26} .
\end{aligned}
$$

Gelfand-Kirillov dimension: 26.

Bernstein degree: $3003-4719+2652-273-273+78=468$. $a=2$ :

Stratum: $(v, T)=\left(v_{25},\left\{\alpha_{4}\right\}\right) \quad(\lambda+\rho$ semi-regular $) \quad$ Vertex: $2 \omega_{6}-12 \omega_{7}$.

Reduced root system: $\Delta_{\lambda} \simeq S U(1,5) ., \Delta_{\lambda, n}^{+}=\left\{\beta_{2}, \beta_{4}, \beta_{7}, \beta_{11}, \beta_{14}\right\}$.

Resolution:

$$
0 \rightarrow N_{5} \stackrel{+2}{\longrightarrow} N_{4} \stackrel{+1}{\longrightarrow} N_{3} \stackrel{+2}{\longrightarrow} N_{2} \stackrel{+1}{\longrightarrow} N_{1} \stackrel{+1}{\longrightarrow} N_{0} \rightarrow L \rightarrow 0 .
$$




$$
\begin{aligned}
& \eta_{0}=\lambda=2 \omega_{6}-12 \omega_{7}, \\
& \eta_{1}=\lambda-\beta_{14}=\omega_{1}+\omega_{6}-12 \omega_{7}, \\
& \eta_{2}=\lambda-\beta_{14}-\beta_{11}=\omega_{3}-12 \omega_{7}, \\
& \eta_{3}=s_{4} s_{6}\left(\lambda+\rho-\beta_{14}-\beta_{11}-2 \beta_{7}\right)-\rho=\omega_{2}-13 \omega_{7}, \\
& \eta_{4}=s_{4} s_{6}\left(\lambda+\rho-\beta_{14}-\beta_{11}-2 \beta_{7}-\beta_{4}\right)-\rho=\omega_{6}-14 \omega_{7}, \\
& \eta_{5}=s_{3} s_{5} s_{4} s_{6}\left(\lambda+\rho-\beta_{14}-\beta_{11}-\cdots-2 \beta_{2}\right)-\rho=-14 \omega_{7} .
\end{aligned}
$$

Hilbert series:

$$
\begin{aligned}
h(t) & =\left(351-650 t+351 t^{2}-78 t^{4}+27 t^{5}-t^{7}\right) /(1-t)^{27} \\
& =\left(351-299 t+52 t^{2}+52 t^{3}-26 t^{4}+t^{5}+t^{6}\right) /(1-t)^{26} .
\end{aligned}
$$

Gelfand-Kirillov dimension: 26 .

Bernstein degree: $351-299+52+52-26+1+1=132$.

$a=1$ :

Stratum: $(v, T)=\left(v_{32},\left\{\alpha_{2}, \alpha_{5}\right\}\right) \quad\left(A_{1} \times A_{1}\right.$ singularity $) \quad$ Vertex: $\omega_{6}-10 \omega_{7}$.

Reduced root system: $\Delta_{\lambda} \simeq S U(1,1), \Delta_{\lambda, n}^{+}=\left\{\beta_{14}\right\}$.

Resolution:

$$
0 \rightarrow N_{1} \stackrel{+1}{\longrightarrow} N_{0} \rightarrow L \rightarrow 0 .
$$

$\eta_{0}=\lambda=\omega_{6}-10 \omega_{7}$,

$\eta_{1}=\lambda-\beta_{3}=\omega_{1}-10 \omega_{7}$.

Hilbert series: $h(t)=(27-27 t) /(1-t)^{27}=27 /(1-t)^{26}$.

Gelfand-Kirillov dimension: 26.

Bernstein degree: 27.

Special feature: $\lambda+\rho$ is quasi-dominant (as defined in [EHW, 4.4]).

7.11. $Q_{\lambda} \simeq E_{7}, z=9$ (first reduction point).

Highest weight: $\lambda=-8 \omega_{7}$.

Stratum: $(v, T)=\left(v_{37},\left\{\alpha_{4}, \alpha_{6}\right\}\right) \quad\left(A_{1} \times A_{1}\right.$ singularity $) \quad$ Vertex $-8 \omega_{7}$.

Reduced root system: $\Delta_{\lambda} \simeq S U(1,1), \Delta_{\lambda, n}^{+}=\left\{\beta_{9}\right\}$.

Resolution:

$$
N_{1} \stackrel{+3}{\longrightarrow} N_{0} \rightarrow L \rightarrow 0
$$

Hilbert series: $h(t)=\left(1-t^{3}\right) / t^{27}=\left(1+t+t^{2}\right) /(1-t)^{26}$.

Gelfand-Kirillov dimension: 26 .

Bernstein degree: 3 .

Special feature: $\lambda+\rho$ is quasi-dominant (Wallach representation).

7.12. $Q_{\lambda} \simeq E_{7}, z=13$ (second reduction point).

Highest weight: $\lambda=-4 \omega_{7}$.

$\lambda+\rho$ semi-regular with singular $\operatorname{root} \beta_{23}$.

Stratum: $(v, T)=\left(v_{52},\left\{\alpha_{4}\right\}\right) \quad(\lambda+\rho$ semi-regular $) \quad$ Vertex: $-4 \omega_{7}$.

Reduced root system: $\Delta_{\lambda} \simeq S O(2,10)$,

$$
\Delta_{\lambda, n}^{+}=\left\{\beta_{0}, \beta_{1}, \beta_{3}, \beta_{4}, \beta_{6}, \beta_{9}, \beta_{10}, \beta_{12}, \beta_{17}, \beta_{20}\right\} \text {. }
$$

Resolution:

$$
\begin{aligned}
0 \rightarrow N_{10} \stackrel{+2}{\longrightarrow} N_{9} \stackrel{+1}{\longrightarrow} N_{8} \stackrel{+2}{\longrightarrow} N_{7} \stackrel{+1}{\longrightarrow} N_{6} \stackrel{(+1,+2)}{\longrightarrow} N_{5}^{\prime} \oplus N_{5}^{\prime \prime} \stackrel{(+2,+1)}{\longrightarrow} \\
N_{4} \stackrel{+1}{\longrightarrow} N_{3} \stackrel{+2}{\longrightarrow} N_{2} \stackrel{+1}{\longrightarrow} N_{1} \stackrel{+2}{\longrightarrow} N_{0} \rightarrow L \rightarrow 0 .
\end{aligned}
$$


Hilbert series:

$$
\begin{aligned}
h(t)= & \left(1-27 t^{2}+78 t^{3}-351 t^{5}+650 t^{6}-351 t^{7}-351 t^{8}\right. \\
& \left.+650 t^{9}-351 t^{10}+78 t^{12}-27 t^{13}+t^{15}\right) /(1-t)^{27} \\
= & \left(1+10 t+28 t^{2}+28 t^{3}+10 t^{4}+t^{5}\right) /(1-t)^{17} .
\end{aligned}
$$

Gelfand-Kirillov dimension: 17 .

Bernstein degree: 78 .

Special feature: $\lambda+\rho$ is quasi-dominant (Wallach representation).

\section{REFERENCES}

[BGG] Bernstein, I. N., Gelfand I. M. and Gelfand, S. I., Differential equations on the base affine space and a study of $\mathfrak{g}$-modules, Lie Groups and their Representations, Summer School of the Bolyai János Math. Soc. edited by I. M. Gelfand, Halsted Press, Division of John Wiley \& Sons, New York, 1975, 21-64.

[C] Collingwood, D., The $\mathfrak{n}$-homology of Harish-Chandra modules: generalizing a theorem of Kostant, Math. Ann. 272 (1985). MR 87a:22027

[DES] Davidson, M. G., Enright, T. J. and Stanke, R. J., Differential operators and highest weight representations, Memoirs AMS 94 (1991), 161-187. MR 92c:22034

[E] Enright, T.J., Analogues of Kostant's u-cohomology formulas for unitary highest weight modules, J. Reine Angew. Math. 392 (1988), 27-36. MR 89m:22022

[EHW] Enright, T. J., Howe, R. and Wallach, N. R., A classification of unitary highest weight modules, Representation theory of reductive groups (Park City, Utah, 1982), Progr. Math. 40 (1983), 97-143. MR 86c:22028

[EH] Enright, T. J. and Hunziker, M., Resolutions and Hilbert series of determinantal varieties and unitary highest weight modules, to appear in J. Algebra.

[EJ] Enright, T. J. and Joseph, A., An intrinsic analysis of unitary highest weight modules, Math. Ann. 288 (1990), 571-594. MR 91m:17005

[ES1] Enright, T. J. and Shelton, B., Categories of highest weight modules: applications to classical Hermitian symmetric pairs, Memoirs AMS 67 (1987). MR 88f:22052

[ES2] Highest weight modules for Hermitian symmetric pairs of exceptional type, Proc. AMS 106 (1989), 807-819. MR 89m:17010

[EW] Enright, T. J. and Willenbring, J.F., Hilbert series, Howe duality and branching for classical groups, to appear in Math. Ann.

[L] Lepowsky, J., A generalization of the Bernstein-Gelfand-Gelfand resolution, J. Algebra 49 (1977), 496-511. MR 57:16367

[NOTYK] Nishiyama, K., Ochiai, H., Taniguchi, K., Yamashita, H., Kato, S., Nilpotent orbits, associated cycles and Whittaker models for highest weigh representations, Asterisque 273 (2001). MR 2002b:22025

[W] Wallach, N. R., The analytic continuation of the discrete series I, II, Trans. Amer. Math. Soc. 251 (1979), 1-17, 19-37. MR 81a:22009

Department of Mathematics, University of California at San Diego, La Jolla, CalIFORNIA 92093-0112

E-mail address: tenright@math.ucsd.edu

Department of Mathematics, University of Georgia, Athens, Georgia 30602-7403

E-mail address: hunziker@math.uga.edu 